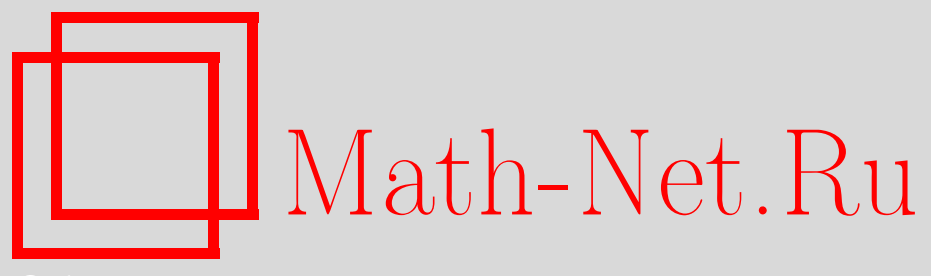

А. Я. Хелемский, Квантовые версии векторной двойственности и экспоненциального закона в рамках безматричного подхода, Матем. сб., 2006, том 197, номер 12, 133-156

DOI: https://doi.org/10.4213/sm1435

Использование Общероссийского математического портала Math-Net.Ru подразумевает, что вы прочитали и согласны с пользовательским соглашением http://www . mathnet.ru/rus/agreement

Параметры загрузки:

IP : 3.80 .181 .102

26 апреля 2023 г., 18:32:27

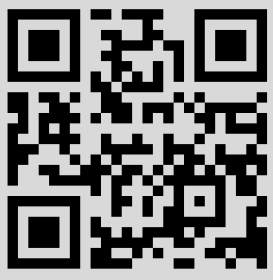




\title{
А. Я. Хелемский \\ Квантовые версии векторной двойственности и экспоненциального закона в рамках безматричного подхода
}

\begin{abstract}
В статье показано, какой вид принимают некоторые принципиальные конструкции и результаты квантового функционального анализа (т.е. теории операторных пространств) в рамках подхода, использующего векторы с операторными коэффициентами вместо матриц. Речь идет о “безматричном" квантовании пространств, находящихся в отношении векторной двойственности. После описания основной конструкции установлены, в рамках "безматричного" подхода, квантовая версия экспоненциального закона и квантовая версия закона сопряженной ассоциативности, связывающего функторы операторов с функторами тензорного произведения. В конце работы в качестве важного конкретного примера рассмотрено "безматричное" квантование алгебры операторов в гильбертовом пространстве и его основные свойства.
\end{abstract}

Библиография: 15 названий.

Настоящая статья посвящена некоторым вопросам квантового функционального анализа - области математики, изучающей так называемые операторные, или квантовые, пространства, алгебры и модули (ср. памятную лекцию Эффpoca [1]). Речь идет об алгебраических объектах, наделенных дополнительной структурой, более богатой, чем обычная норма. В подавляющем большинстве работ (особо выделим монографии [2]-[4], по которым уже можно учиться) эта структура задается путем одновременного введения нормы в каждое из пространств матриц всех размерностей с коэффициентами из данного объекта.

Есть, однако, и другой путь. Вместо последовательности норм в пространствах матриц можно рассмотреть одну норму, но в более сложном пространстве, чем матричные. Это пространство состоит, говоря неформально, из векторов исходного линейного пространства с коэффициентами из какой-либо достаточно богатой операторной алгебры. Подобная замена скаляров операторами, на этот раз в качестве коэффициентов при наших векторах, вполне соответствует общей философии “квантовой”, или некоммутативной математики.

Тот факт, что оба подхода, матричный (координатный) и "операторный" (бескоординатный), дают по существу одни и те же результаты, известен.

Это хорошо понимал, судя по его неопубликованным запискам, Барри Джонсон. Об этом же сказано в книге Пизье [5], в которой, несмотря на явное преобладание матричного подхода, есть несколько красивых выходов в "бескоординатную" теорию (ср. дальнейшие ссылки в основном тексте статьи). Наконец,

Работа выполнена при поддержке Российского фонда фундаментальных исследований (грант № 05-01-00982).

(С) А. Я. ХЕлЕмский, 2006 
равнозначность обоих подходов, в виде теорем об эквивалентности различного рода категорий, показана в работах [6], [7]; ср. также результаты о реализации операторных бимодулей в [8], [9].

И все же, какую конкретную форму приобретают основные конструкции и результаты квантового функционального анализа именно в рамках бескоординатного подхода, без оглядки на матрицы? И что появляется нового? Похоже на то, что систематического изложения теории с указанных позиций еще не предлагалось.

В этой статье мы хотим показать, какой вид принимают некоторые принципиальные конструкции и результаты квантового функционального анализа в рамках подхода, использующего векторы с операторными коэффициентами вместо матриц. Речь пойдет о квантовании пространств, находящихся в отношении двойственности - как скалярной, так и векторной - и, в частности, о квантовании пространств полностью ограниченных операторов между квантовыми пространствами. После рассказа об основной конструкции будут установлены квантовая версия экспоненциального закона классического функционального анализа и квантовая версия закона сопряженной ассоциативности, связывающего функторы операторов с функторами тензорного произведения.

\section{§ 1. Предварительные определения и сведения}

Ради краткости, говоря "оператор", мы будем всегда подразумевать линейный оператор, а говоря "биоператор" - билинейный оператор. Аналогичный смысл мы придадим словам "функционал" и "бифункционал".

Если $E$ и $F$ - нормированные пространства, то мы, как обычно, обозначаем через $\mathscr{B}(E, F)$ пространство всех ограниченных, а через $\mathscr{K}(E, F)$ - пространство всех компактных операторов из $E$ в $F$, и пишем $\mathscr{B}(E)$ вместо $\mathscr{B}(E, E)$ и $\mathscr{K}(E)$ вместо $\mathscr{K}(E, E)$. Если $G$ - еще одно нормированное пространство, то мы обозначаем через $\mathscr{B}(E \times F, G)$ пространство всех ограниченных биоператоров из $E \times F$ в $G$. Все эти пространства рассмотрены с (би)операторной нормой.

Для гильбертова тензорного произведения гильбертовых пространств, равно как и для гильбертова тензорного произведения действующих в них операторов, мы будем употреблять знак $\dot{\otimes}$.

Выберем какое-либо сепарабельное бесконечномерное гильбертово пространство, обозначим его через $L$ и зафиксируем его на протяжении всей статьи. Тождественный оператор в $L$ мы обозначим через 1. Для операторных алгебр $\mathscr{B}(L)$ и $\mathscr{K}(L)$ примем краткие обозначения $\mathscr{B}$ и $\mathscr{K}$. Далее, зафиксируем опять-таки, "навсегда" - какой-либо изометрический (равносильно, унитарный) изоморфизм $\iota: L \rightarrow L \dot{\otimes} L$ и положим $\varsigma:=\mathscr{B}(L \dot{\otimes} L) \rightarrow \mathscr{B}: a \mapsto \iota^{*} a \iota$. Разумеется, $\varsigma$ - изометрический *-изоморфизм операторных алгебр. Для действующего в $L$ оператора $\varsigma(a \dot{\otimes} b), a, b \in \mathscr{B}$, мы условимся употреблять краткое обозначение $a \diamond b$. (Это "бубновое" умножение по своим свойством напоминает тензорное, но в отличие от последнего не выводит за пределы $\mathscr{B}$.)

В рассматриваемом контексте возникает некоторый унитарный оператор периода 2 , действующий в $L$. Он вводится с помощью унитарного оператора переброса ("flip") $\nabla: L \dot{\otimes} L \rightarrow L \dot{\otimes} L$, корректно определенного правилом $\xi \otimes \eta \mapsto \eta \otimes \xi$, 
$\xi, \eta \in L$. А именно, таков, как легко видеть, $\triangle:=\iota^{*} \nabla \iota($ он же и $\varsigma(\nabla))$. Обратим внимание на очевидное равенство

$$
b \diamond a=\triangle(a \diamond b) \triangle .
$$

Пусть $X$ - (пока произвольный) унитальный бимодуль над алгеброй $\mathscr{B}$. Носителем элемента $u \in X$ мы назовем любой (подразумевается: ортогональный) проектор $P \in \mathscr{B}$ такой, что $P \cdot u \cdot P=u$. Разумеется, если $\varphi: X \rightarrow Y-$ морфизм $\mathscr{B}$-бимодулей, то всякий носитель элемента $u \in X$ является и носителем элемента $\varphi(u) \in Y$.

Заметим также, что наш $X$ наряду с заданной $\mathscr{B}$-бимодульной структурой обладает еще двумя. Первая вводится с помощью равенств $a \cdot 1 u:=(a \diamond \mathbf{1}) \cdot u$ и $u \cdot{ }_{1} a:=u \cdot(a \diamond \mathbf{1})$, а вторая - с помощью равенств $a \cdot 2 u:=(\mathbf{1} \diamond a) \cdot u$ и $u \cdot \cdot_{2} a:=u \cdot(\mathbf{1} \diamond a)$. Все нужные свойства легко проверяются.

Пусть $\|\cdot\|$ - заданная в $X$ норма. Мы будем говорить, что эта норма удовлетворяет первой аксиоме Руана, или аксиоме (RI), если для любых $a \in \mathscr{B}$ и $u \in X$ выполнено $\|a \cdot u\|,\|u \cdot a\| \leqslant\|a\|\|u\|$. (В терминологии теории банаховых алгебр это означает, что $X$ является сжимающим нормированным $\mathscr{B}$ бимодулем.) Прежде чем двигаться дальше, отметим очевидное

ПреДЛОЖЕНИЕ 1. Пусть норма в $X$ удовлетворяет аксиоме $(\mathrm{RI}), x \in X$, $a$ - изометрический, $b$ - коизометрический операторь в L. Тогда

$$
\|a \cdot x\|=\|x \cdot b\|=\|x\| .
$$

Далее, мы будем говорить, что норма в $X$ удовлетворяет второй аксиоме $P y$ $a н a$, или аксиоме (RII), если для любых $u, v \in X$, обладающих ортогональными носителями, выполнено $\|u+v\|=\max \{\|u\|,\|v\|\}$.

Выделим для удобства будущих ссылок три простых утверждения. Во всех трех мы предполагаем, что норма в $X$ удовлетворяет аксиомам Руана относительно внешних умножений, обозначенных ". ".

ПреДЛОжениЕ 2. Эта же норма удовлетворяет аксиомам Руана и относительно внешних умножений ". 1 " $u$ ". 2 ".

ПреДЛОЖЕНИЕ 3. Пусть Е - нормированное пространство. Тогда в про-

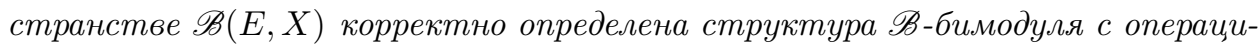
ями $[a \cdot \varphi](u):=a \cdot \varphi(u) u[\varphi \cdot a](u):=\varphi(u) \cdot a, a \in \mathscr{B}, \varphi \in \mathscr{B}(E, X), u \in X$. При этом операторная норма в $\mathscr{B}(E, X)$ также удовлетворяет аксиомам Руана.

ПреДЛОЖЕНИЕ 4. Пусть $Y$ - еще один $\mathscr{B}$-бимодуль и $\varphi: Y \rightarrow X-$ ингективный морфизм Я̈-бимодулей. Тогда $Y$, снабженный нормой $\|v\|:=\|\varphi(v)\|$, также удовлетворяет аксиомам Руана.

Мы переходим к основным понятиям квантового функционального анализа. Для линейного пространства $E$ алгебраическое тензорное произведение $\mathscr{K} \otimes E$ мы будем называть размножением $E$ и кратко обозначать $\mathscr{K} E$. (Его удобно мыслить как "пространство формальных линейных комбинаций векторов из $E$ с соответствующими операторными коэффициентами".) Кроме того, его элементарные тензоры мы будем обозначать через $а x$ вместо $a \otimes x, a \in \mathscr{K}, x \in E$. 
Пространство $\mathscr{K}$ м мы будем рассматривать как бимодуль над $\mathscr{B}$ относительно внешних умножений, корректно определенных равенствами $a \cdot b x=(a b) x$ и $b x \cdot a=(b a) x, a \in \mathscr{B}, b \in \mathscr{K}, x \in E$.

Квантовая норма в $E$ - это по определению норма в $\mathscr{B}$-бимодуле $\mathscr{K} E$, удовлетворяющая обеим аксиомам Руана. Пространство, снабженное квантовой нормой, называется квантовым нормированным пространством или просто квантовым пространством.

Квантовое нормированное пространство становится "классическим" нормированным пространством, если для $x \in E$ положить $\|x\|:=\|p x\|$, где $p$ - произвольный одномерный проектор из $\mathscr{B}$. Из аксиомы (RI) легко следует, что это действительно норма, не зависящая от выбора $p$. (На самом деле, мы могли бы вместо $p x$ взять $a x$, где $a$ - любой компактный оператор нормы 1 , но этот факт нам не понадобится.) Полученный объект называется подлежащим нормированным пространством исходного квантового и иногда, во избежание путаницы, обозначается $\square E$. Само же исходное квантовое нормированное пространство $E$ мы будем называть квантованием своего подлежащего пространства $\square E$, а его квантовую норму - квантованием нормы в $\square E$. Подчеркнем, что одно и то же нормированное пространство имеет, вообще говоря, много весьма несхожих квантований. Однако простейшее нормированное пространство - комплексная плоскость - обладает, как легко следует из аксиом Руана, единственным квантованием. А именно, норма в $\mathscr{K} \mathbb{C}=\mathscr{K}$, являющаяся квантованием нормы в $\mathbb{C}$, - это обычная операторная норма.

Везде далее, говоря о (просто) норме элемента квантового пространства $E$, мы будем иметь в виду норму этого элемента в $\square E$.

Если $F$ - подпространство в квантовом пространстве $E$, то оно само является квантовым пространством относительно квантовой нормы, определенной равенством $\|u\|:=\left\|\left(\mathbf{1}_{\mathscr{K}} \otimes i\right)(u)\right\|$, где $i: F \rightarrow E-$ естественное вложение. (Это частный случай предложения 4.) В такой ситуации мы будем говорить о соответствующем квантовом подпространстве.

Укажем весьма общий класс примеров квантовых пространств. Пусть нормированное пространство $E$ задано в явном виде как нормированное подпространство в $\mathscr{B}(H)$, где $H$ - некоторое гильбертово пространство, и $j_{E}: E \rightarrow$ $\mathscr{B}(H)$ - естественное вложение. Рассмотрим оператор $\widehat{\jmath}_{E}: \mathscr{K} E \rightarrow \mathscr{B}(L \dot{\otimes} H)$, ассоциированный с биоператором $\mathscr{K} \times E \rightarrow \mathscr{B}(L \dot{\otimes} H):(a, x) \mapsto a \dot{\otimes} x$. Этот оператор, как легко видеть, инъективен и, таким образом, позволяет отождествить $\mathscr{K} E$ с подпространством в $\mathscr{B}(L \dot{\otimes} H)$. (Он же является копродолжением на $\mathscr{B}(L \dot{\otimes} H)$ оператора $\mathbf{1}_{\mathscr{K}} \otimes j_{E}: \mathscr{K} E \rightarrow \mathscr{K} \mathscr{B}(H)$.) Тогда, задав в $\mathscr{K} E$ норму, индуцированную этим оператором, мы, как легко видеть, получим квантовую норму в $E$. Эта квантовая норма, а также соответствующие квантовое пространство и квантование называются конкретными.

ЗАмечание 1. Ныне знаменитая теорема Руана, или, вернее, ее надлежащая "бескоординатная" версия, утверждает, что любое ("абстрактное") квантовое пространство совпадает, с точностью до определяемого нами чуть ниже полностью изометрического изоморфизма, с некоторым конкретным. Но круг вопросов, связанный с этой теоремой, в настоящей статье не рассматривается.

Вернемся к общим квантовым пространствам. 
ПреДЛОЖЕНИЕ 5. Пусть $d_{n}, n=1,2, \ldots,-$ аппроксимативная единица в $\mathscr{K}$. Тогда $\left\|d_{n} \cdot u\right\|,\left\|u \cdot d_{n}\right\| \rightarrow\|u\|, n \rightarrow \infty$, для любого $u \in \mathscr{K} E$.

ДокАЗАТЕЛьство. Соображения линейности позволяют ограничиться случаем $u=a x, a \in \mathscr{K}, x \in E$. Как известно, $a$ представим как произведение $b c$ двух других компактных операторов. Тогда $a x-d_{n} \cdot a x=\left(b-d_{n} b\right) \cdot c x$ и $a x-a x \cdot d_{n}=b x \cdot\left(c-c d_{n}\right)$. Поэтому аксиома $(\mathrm{RI})$ влечет

$$
\left\|a x-d_{n} \cdot a x\right\|,\left\|a x-a x \cdot d_{n}\right\| \rightarrow 0, \quad n \rightarrow \infty .
$$

Дальше ясно.

ПРЕДЛОЖЕНИЕ 6. Для любого элементарного тензора в КЕ выполнена оценка $\|$ ax $\|\leqslant\| a\|\| x \|$.

ДокАЗАТЕЛьство. Пусть $e_{k}, k=1,2, \ldots,-$ ортонормированный базис в $L$, $p_{k}$ - проектор на $\operatorname{span}\left\{e_{k}\right\}$ и $P_{n}:=\sum_{k=1}^{n} p_{k}$. В силу $a x \cdot P_{n}=\left(a P_{n}\right) x=a \cdot P_{n} x$ из предыдущего предложения и аксиомы (RI) следует, что

$$
\|a x\|=\lim _{n \rightarrow \infty}\left\|a \cdot P_{n} x\right\| \leqslant \varlimsup_{n \rightarrow \infty}\|a\|\left\|P_{n} x\right\| .
$$

Но $P_{n} x=\sum_{k=1}^{n} p_{k} x$, а элементы $p_{k} x$ обладают попарно ортогональными носителями, а именно $p_{k}$. Поэтому из аксиомы (RII) очевидным образом следует, что $\left\|P_{n} x\right\|=\max \left\{\left\|p_{k} x\right\|: k=1, \ldots, n\right\}$. Но каждое из чисел $\left\|p_{k} x\right\|$ есть $\|x\|$. Дальше ясно.

ЗАмечАниЕ 2. На самом деле, как нетрудно показать (используя разложение компактных операторов в ряд Шмидта), выполнено точное равенство $\|a x\|=\|a\|\|x\|$. Но этот факт нам не понадобится.

Укажем важную для дальнейшего общую конструкцию.

Сперва рассмотрим "вторичное размножение" $\mathscr{K}[\mathscr{K E}]$ линейного пространства $E$ и оператор $\varsigma_{E}: \mathscr{K}[\mathscr{K} E] \rightarrow \mathscr{K} E$, ассоциированный с 3 -линейным оператором $\mathscr{K} \times \mathscr{K} \times E \rightarrow \mathscr{K} E:(a, b, x) \mapsto(a \diamond b) x$. Таким образом, этот оператор корректно определен правилом $a[b x] \mapsto(a \diamond b) x$. Он же, очевидно, является биограничением на соответствующие пространства оператора $\varsigma \otimes \mathbf{1}_{E}:[\mathscr{B}(L \dot{\otimes} L)] \otimes E \rightarrow$ $\mathscr{B} \otimes E$. Легко проверить, что $\varsigma_{E}-$ инъективный морфизм $\mathscr{B}$-бимодулей относительно внешних умножений в $\mathscr{K}[\mathscr{K} E]$ (как в размножении пространства $\mathscr{K} E)$ и внешних умножений ".. " в $\mathscr{K} E$ (см. выше).

Если наше $E$ - квантовое пространство, то для $u \in \mathscr{K}[\mathscr{K} E]$ мы положим $\|u\|:=\left\|\varsigma_{E}(u)\right\|$. Объединяя только что сделанное наблюдение с предложениями 2 и 4, мы видим, что это равенство определяет норму в $\mathscr{K}[\mathscr{K} E]$, удовлетворяющую обеим аксиомам Руана. Далее, если $e-$ вектор в $L$ нормы $1, p \in \mathscr{K}-$ проектор на $\operatorname{span}\{e\}$ и оператор $\rho: L \rightarrow L \dot{\otimes} L$ действует по правилу $\xi \mapsto e \otimes \xi$, то, как легко видеть, $\rho a \rho^{*}=p \dot{\otimes} a$ для всех $a \in \mathscr{B}$. Поэтому для элементарного тензора в $\mathscr{K} E$, скажем $a x$, выполнено равенство

$$
\varsigma_{E}(p[a x])=(\varsigma(p \dot{\otimes} a)) x=\left[\iota^{*}(p \dot{\otimes} a) \iota\right] x=\left(\iota^{*} \rho a \rho^{*} \iota\right) x=\left(\iota^{*} \rho\right) \cdot(a x) \cdot\left(\rho^{*} \iota\right) .
$$

Отсюда по линейности $\varsigma_{E}(p u)=\left(\iota^{*} \rho\right) \cdot u \cdot\left(\rho^{*} \iota\right)$ для любого $u \in \mathscr{K} E$. Поскольку оператор $\iota^{*} \rho$ изометрический, а $\rho^{*} \iota$ коизометрический, предложение 1 дает 
$\|p u\|=\left\|\varsigma_{E}(p u)\right\|=\|u\|$. Этим показано, что построенная норма в $\mathscr{K}[\mathscr{K E}]$ является квантованием исходной нормы в $\mathscr{K} E$ (равносильно, квантовой нормы в $E$ ). Такую квантовую норму в $\mathscr{K} E$ мы будем (рискуя навлечь праведный гнев физиков) называть вторичным квантованием квантовой нормы в $Е$.

Забыв на время о бимодульной структуре в $\mathscr{K}[\mathscr{K} E]$, можно заметить, что это пространство - оно же $\mathscr{K} \otimes \mathscr{K} \otimes E$ - обладает некоей внутренней симметрией, доставляемой "оператором рокировки" $\mho_{E}: \mathscr{K}[\mathscr{K} E] \rightarrow \mathscr{K}[\mathscr{K} E]$, корректно определенным равенством $\mho_{E}(a[b x])=b[a x], a, b \in \mathscr{K}$. Этот же оператор может быть определен и с помощью оператора $\nabla: L \dot{\otimes} L \rightarrow L \dot{\otimes} L$, определенного выше. А именно, если положить $\widetilde{\mho}: \mathscr{B}(L \dot{\otimes} L) \rightarrow \mathscr{B}(L \dot{\otimes} L): a \mapsto \nabla a \nabla, a \in L \dot{\otimes} L$, то $\mho_{E}$, как легко видеть, есть сужение оператора $\widetilde{\mho} \otimes \mathbf{1}_{E}: \mathscr{B}(L \dot{\otimes} L) \otimes E \rightarrow \mathscr{B}(L \dot{\otimes} L) \otimes E$ на его инвариантное подпространство $\mathscr{K}[\mathscr{K} E]$.

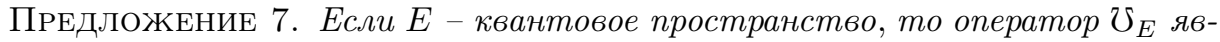
ляется изометрическим изоморфизмом относительно вторичного квантования квантовой нормы в Е.

ДокАЗАтЕльство. Напомним об обсуждавшемся выше операторе $\triangle: L \rightarrow L$. В силу (1) для любых $a, b \in \mathscr{K}, x \in E$ выполнено равенство

$$
\varsigma_{E}\left(\mho_{E}(a[b x])\right)=\varsigma_{E}(b[a x])=(b \diamond a) x=[\triangle(a \diamond b) \triangle] x=\triangle \cdot \varsigma_{E}(a[b x]) \cdot \triangle .
$$

Отсюда по линейности $\varsigma_{E}\left(\mho_{E}(u)\right)=\triangle \cdot \varsigma_{E}(u) \cdot \triangle$ для всех $u \in \mathscr{K}[\mathscr{K} E]$. В силу предложения 1 это влечет $\left\|\varsigma_{E}\left(\mho_{E}(u)\right)\right\|=\left\|\varsigma_{E}(u)\right\|$. Дальше ясно.

Пусть теперь $\varphi: E \rightarrow F$ - оператор между двумя линейными пространствами. Морфизм $\mathscr{B}$-бимодулей $\mathbf{1}_{\mathscr{K}} \otimes \varphi: \mathscr{K} E \rightarrow \mathscr{K} F$ мы будем называть размножением этого оператора и кратко обозначать $\varphi_{\infty}$. Если $E$ и $F-$ квантовые пространства, то оператор $\varphi$ называется полностъю ограниченным, если его размножение - ограниченный (в "классическом" смысле) оператор. Операторная норма $\left\|\varphi_{\infty}\right\|$ обозначается также $\|\varphi\|_{c b}$ и называется полностью ограниченной нормой (исходного) оператора $\varphi$. Как легко видеть, множество $\mathscr{C} \mathscr{B}(E, F)$, состоящее из полностью ограниченных операторов между $E$ и $F$, является нормированным пространством относительно поточечных операций и нормы $\|\varphi\|_{c b}$.

Разумеется, если $\varphi$ полностью ограничен, то он ограничен и как оператор между соответствующими подлежащими пространствами (т.е. между $\square E$ и $\square F)$, и при этом $\|\varphi\| \leqslant\|\varphi\|_{c b}$. (Важно, что обратное, вообще говоря, неверно; cp. [10].) Таким образом, пространство $\mathscr{C} \mathscr{B}(E, F)$ является линейным подпространством в $\mathscr{B}(E, F)$ (вообще говоря, не замкнутым относительно операторной нормы).

Оператор между двумя квантовыми пространствами называется полностъю изометрическим (соответственно полностью коизометрическим), если его размножение - изометрический (соответственно коизометрический) оператор, и полностъю изометрическим изоморфизмом, если его размножение изометрический изоморфизм.

Приведем важный для дальнейшего пример. 
ПреДЛОЖЕНИЕ 8. Пусть Е - квантовое пространство, $\mathscr{K}$ и и $\mathscr{K}[\mathscr{K E}]-$ квантовые пространства, полученные с помощъю его вторичного и "третичного" (в очевидном смысле) квантования. Тогда $\varsigma_{E}: \mathscr{K}[\mathscr{K} E] \rightarrow \mathscr{K} E-$ полностью изометрический оператор.

ДоказАтельство. Рассмотрим цепочку операторов

$$
L \stackrel{\iota}{\longrightarrow} L \dot{\otimes} L \stackrel{\iota \dot{\otimes} \mathbf{1}}{\longrightarrow} L \dot{\otimes} L \dot{\otimes} L \stackrel{1 \dot{\otimes} \iota^{*}}{\longrightarrow} L \dot{\otimes} L \stackrel{\iota^{*}}{\longrightarrow} L
$$

и обозначим их композицию через $I: L \rightarrow L$. Ясно, что $I-$ изометрический изоморфизм и при этом, как легко проверить, $I((a \diamond b) \diamond c) I^{*}=a \diamond(b \diamond c)$ для Bcex $a, b, c \in \mathscr{B}$.

А теперь рассмотрим диаграмму

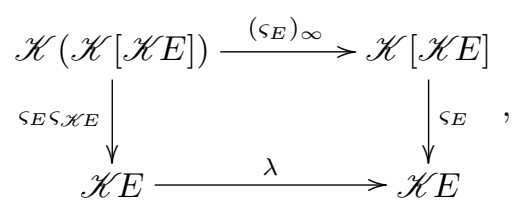

в которой $\lambda$ переводит элемент $u$ в $I \cdot u \cdot I^{*}$. Взяв элементарный тензор $a(b[c x]) \in$ $\mathscr{K}(\mathscr{K}[\mathscr{K} E])$ и “пойдя вниз", мы, очевидно, получим элемент $((a \diamond b) \diamond c) x$, a "пойдя вправо и вниз", мы, очевидно, получим $(a \diamond(b \diamond c)) x$. Отсюда немедленно следует, что наша диаграмма коммутативна. Но в силу предложения $1 \lambda-$ изометрический изоморфизм, а в силу определения вторичного квантования вертикальные стрелки изображают изометрические операторы. Это означает, что $\left(\varsigma_{E}\right)_{\infty}$ - также изометрический оператор. Дальше ясно.

Наконец, пусть $E, F$ и $G$ - три линейных пространства, а $\mathscr{R}: E \times F \rightarrow G-$ биоператор. Слабым размножением последнего называется биоператор $\mathscr{R}_{w}$ : $\mathscr{K} E \times \mathscr{K} F \rightarrow \mathscr{K} G$, ассоциированный с 4-линейным оператором $\mathscr{K} \times E \times \mathscr{K} \times F \rightarrow$ $\mathscr{K} G:(a, x, b, y) \mapsto(a \diamond b) \mathscr{R}(x, y)$. Таким образом, $\mathscr{R}_{w}$ однозначно определен равенством $\mathscr{R}_{w}(a x, b y)=(a \diamond b) \mathscr{R}(x, y)$. Если речь идет о квантовых пространствах, то наш биоператор называется слабо полностъю ограниченным, если его слабое размножение - ограниченный (опять-таки в классическом смысле) биоператор. (Ср. "матричное" определение в [2; с. 126].) Биоператорная норма $\left\|\mathscr{R}_{w}\right\|$ обозначается также $\|\mathscr{R}\|_{w c b}$ и называется слабо полностъю ограниченной нормой (исходного) оператора $\varphi$. Как легко видеть, множество слабо полностью ограниченных операторов из $E \times F$ в $G$, обозначаемое через $\mathscr{W} \mathscr{B}(E \times F, G)$, является нормированным пространством относительно поточечных операций и нормы $\|\mathscr{R}\|_{w c b}$.

(Есть еще и сильно полностью ограниченные биоператоры, но они в этой статье не рассматриваются; см. [10].)

Если $\mathscr{R}$ слабо полностью ограничен, то он ограничен и как биоператор между соответствующими подлежащими пространствами и при этом $\|\mathscr{R}\| \leqslant$ $\|\mathscr{R}\|_{w c b}$. (Это сразу следует из того очевидного наблюдения, что "бубновый квадрат" одномерного проектора сам является одномерным проектором.) Таким образом, пространство $\mathscr{W} \mathscr{B}(E \times F, G)$ является (вообще говоря, не замкнутым) подпространством в $\mathscr{B}(E \times F, G)$. 
Важный факт, используемый в дальнейшем, состоит в том, что обсуждаемый класс биоператоров допускает линеаризацию с помощью надлежащей "квантовой" версии проективного тензорного произведения нормированных пространств. Точный смысл сказанного таков.

Если Е и F - квантовые пространства, то в алгебраическом тензорном произведении $E \otimes F$ существует однозначно определенная квантовая норма, обозначаемая нами через $\|\cdot\|_{4}$ и обладающая следующим свойством. Пусть $E \otimes_{4} F$ - это пространство $E \otimes F$, рассмотренное с нормой $\|\cdot\|_{4}, G$ - квантовое пространство. Тогда отображсние, сопоставляющее каждому слабо полностъю ограниченному биоператору $\mathscr{R}: E \times F \rightarrow G$ ассочиированный с ним оператор $R: E \otimes F \rightarrow G$ (однозначно определенный правилом $R(x \otimes y)=$ $\mathscr{R}(x, y))$, есть изометрический изоморфизм между нормированными пространствами $\mathscr{W} \mathscr{B}(E \times F, G)$ и $\mathscr{C} \mathscr{B}\left(E \otimes_{4} F, G\right)$.

(Явная конструкция квантовой нормы $\|\cdot\|_{4}$, описанная в [10], нам не понадобится.)

Введенную квантовую норму мы называем четырехименной квантовой нормой, а квантовое пространство $E \otimes_{4} F$ - четьрехименным тензорным произведением квантовых пространств $E$ и $F$. ("Матричные" прототипы этих понятий были открыты четырьмя математиками: Эффросом и Руаном [11] и, независимо и одновременно, Блечером и Полсеном [12].)

ЗАмЕчАниЕ 3. Нормированные пространства, фигурирующие в этой статье, вообще говоря, не предполагаются полными. Мы лишь отметим, что существует содержательное понятие квантового банахова пространства и упомянутое выше четырехименное тензорное произведение имеет свой банахов аналог. Последний строится с помощью надлежащей квантовой версии классической конструкции пополнения нормированного пространства. Но здесь мы касаться этих вопросов не будем.

\section{§ 2. Квантование пространств, находящихся в отношении векторной двойственности}

Пусть снова $E, F$ и $G$ - линейные пространства, $\mathscr{R}: E \times F \rightarrow G$ - биоператор. Как обычно, последний порождает линейные операторы $\mathscr{R}^{F}: F \rightarrow \mathscr{L}(E, G)$ и $\mathscr{R}^{E}: E \rightarrow \mathscr{L}(F, G)$, где $\mathscr{L}(\cdot, \cdot)$ - символ пространства всех линейных операторов. Напомним, что $\mathscr{R}^{F}$ переводит $y \in F$ в оператор $\mathscr{R}^{y}: x \mapsto \mathscr{R}(x, y)$, a $\mathscr{R}^{E}$ переводит $x \in E$ в оператор ' $\mathscr{R}^{x}: y \mapsto \mathscr{R}(x, y)$. (Если операторы $\mathscr{R}^{F}$ и $\mathscr{R}^{E}$ инъективны, то исходный биоператор часто называют векторной двойственностью.) Соответственно, для слабого размножения нашего биоператора возникают операторы $\mathscr{R}_{w}^{\mathscr{K} F}: \mathscr{K} F \rightarrow \mathscr{L}(\mathscr{K} E, \mathscr{K} G): v \mapsto \mathscr{R}_{w}^{v}: u \mapsto \mathscr{R}_{w}(u, v)$ и $\mathscr{R}_{w}^{\mathscr{K} E}: \mathscr{K} E \rightarrow \mathscr{L}(\mathscr{K} F, \mathscr{K} G): u \mapsto \mathscr{R}_{w}^{u}: v \mapsto \mathscr{R}_{w}(u, v)$. Мы видим, в частности, что $\mathscr{R}_{w}^{b y}(a x)={ }^{\prime} \mathscr{R}_{w}^{a x}(b y)=(a \diamond b) \mathscr{R}(x, y)$. (Можно доказать, что если $\mathscr{R}-$ векторная двойственность, то таков же и $\mathscr{R}_{w}$, но нам это не понадобится.)

ТеОРема 1. Пусть $E$ и $G$ - квантовые пространства, $F$ - линейное пространство, а $\mathscr{R}: E \times F \rightarrow G$ - векторная двойственность. Пусть, далее, $\mathscr{R}$ таков, что для любого $y \in F$ оператор $\mathscr{R}^{y}: E \rightarrow G$ полностъю ограничен. 
Тогда F обладает квантовой нормой, корректно определенной равенством

$$
\|v\|:=\sup \left\{\left\|\mathscr{R}_{w}(u, v)\right\|: u \in \mathscr{K} E,\|u\| \leqslant 1\right\} .
$$

При этом (обычная) норма вектора $y \in \square F$ есть $\left\|\mathscr{R}^{y}\right\|_{c b}$.

Мы докажем эту теорему в расширенной формулировке, доставляющей несколько эквивалентных способов введения указанного квантования, а также “симметричное" утверждение о возможности квантования первого аргумента биоператора $\mathscr{R}$. Для этого надо ввести еще несколько операторов, порожденных исходным биоператором.

Наряду с $\mathscr{R}^{y}$ и ' $\mathscr{R}^{x}$ рассмотрим их размножения

$$
\mathscr{R}_{\infty}^{y}: \mathscr{K} E \rightarrow \mathscr{K} G \quad \text { и } \quad ' \mathscr{R}_{\infty}^{x}: \mathscr{K} F \rightarrow \mathscr{K} G .
$$

Для $v \in \mathscr{K} F$ и $u \in \mathscr{K} E$ положим

$$
\widetilde{\mathscr{R}}^{v}: E \rightarrow \mathscr{K} G: x \mapsto \mathscr{R}_{\infty}^{x}(v) \quad \text { и } \quad{ }^{\prime} \widetilde{\mathscr{R}}^{u}: F \rightarrow \mathscr{K} G: y \mapsto \mathscr{R}_{\infty}^{y}(u) .
$$

В частности, с учетом равенств $\mathscr{R}^{y}(x)=\mathscr{R}(x, y)={ }^{\prime} \mathscr{R}^{x}(y)$ для соответствующих элементарных тензоров имеют место формулы

$$
\left[\widetilde{\mathscr{R}}^{b y}\right](x)=b\left[\mathscr{R}^{y}(x)\right] \quad \text { и } \quad\left[{ }^{\prime} \widetilde{\mathscr{R}}^{a x}\right](y)=a\left[\mathscr{R}^{\prime}(y)\right] .
$$

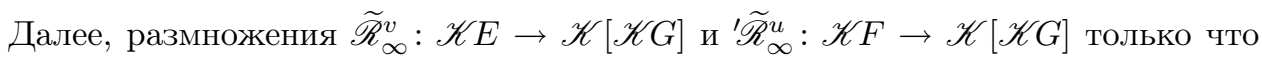
введенных операторов удовлетворяют равенствам

$$
\widetilde{\mathscr{R}}_{\infty}^{b y}(u)=\mho_{G} b\left[\mathscr{R}_{\infty}^{y}(u)\right] \quad \text { и } \quad \widetilde{\mathscr{R}}_{\infty}^{a x}(v)=\mho_{G} a\left[\mathscr{R}_{\infty}^{x}(v)\right],
$$

где $\mho_{G}$ - оператор рокировки из $\S 1$. Оба равенства легко проверяются на элементарных тензорах соответственно в $\mathscr{K} E$ и $\mathscr{K} F$. Отсюда с учетом равенств $\mathscr{R}_{w}^{v}(u)=\mathscr{R}_{w}(u, v)={ }^{\prime} \mathscr{R}_{w}^{u}(v)$, а также (3) вытекают формулы

$$
\mathscr{R}_{w}^{v}(u)=\varsigma_{G}\left(\widetilde{\mathscr{R}}_{\infty}^{v}(u)\right) \quad \text { и } \quad ' \mathscr{R}_{w}^{u}(v)=\varsigma_{G} \mho_{G}\left({ }^{\prime} \widetilde{\mathscr{R}}_{\infty}^{u}(v)\right),
$$

которые также легко проверяются на элементарных тензорах. Наконец, введем в рассмотрение операторы

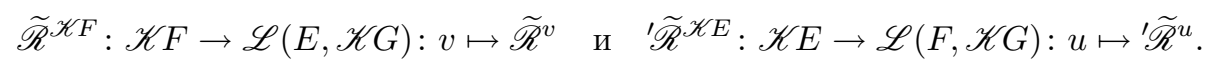

Теорема 1'. Пусть для $E, F, G$ и $\mathscr{R}$ выполнены условия теоремы 1. Тогда (i) оператор $\widetilde{\mathscr{R}}^{\mathscr{K} F}$ принимает значения в $\mathscr{C} \mathscr{B}(E, \mathscr{K} G)$, где квантовая норма в $\mathscr{K} G$ понимается в смысле вторичного квантования;

(ii) оператор $\mathscr{R}_{w}^{\mathscr{K} F}$ принимает значения в $\mathscr{B}(\mathscr{K} E, \mathscr{K} G)$;

(iii) для всех $v \in \mathscr{K} F$ числовое множество $\left\{\left\|\mathscr{R}_{w}(u, v)\right\|: u \in \mathscr{K} E,\|u\| \leqslant 1\right\}$ ограничено;

(iv) (главное) равенство

$$
\|v\|:=\sup \left\{\left\|\mathscr{R}_{w}(u, v)\right\|: u \in \mathscr{K} E,\|u\| \leqslant 1\right\}
$$

задает в F квантовую норму, и эта же квантовая норма удовлетворяет равенствам

$$
\|v\|=\left\|\widetilde{\mathscr{R}}^{v}\right\|_{c b}=\left\|\mathscr{R}_{w}^{v}\right\|
$$


(v) норма вектора $y \in \square F$ есть $\left\|\mathscr{R}^{y}\right\|_{c b}$;

(vi) биоператор $\mathscr{R}$ слабо полностью ограничен относительно исходной квантовой нормы в E и построенной квантовой нормы в F; более того, $\|\mathscr{R}\|_{w c b}=1$.

“Симметричная часть”. Аналогичное утверждение о квантовании первого аргумента нашего биоператора также имеет место. Более подробно, если $E, F, G$ и $\mathscr{R}$ таковы, что для любого $x \in E$ оператор ' $\mathscr{R}^{x}: F \rightarrow G$ полностью ограничен, то сказанное в $n$. (i) верно после замены $\widetilde{\mathscr{R}}^{\mathscr{K}}$ на' $\widetilde{\mathscr{R}}^{\mathscr{K}}$, сказанное в n. (ii) верно после замены $\mathscr{R}_{w}^{\mathscr{K} F}$ на' $\mathscr{R}_{w}^{\mathscr{K}}$ и сказанное в $n$. (iii) верно после замены $v \in \mathscr{K} F$ на $u \in \mathscr{K} E u\left\{\left\|\mathscr{R}_{w}(u, v)\right\|: u \in \mathscr{K} E,\|u\| \leqslant 1\right\}$ на $\left\{\left\|\mathscr{R}_{w}(u, v)\right\|:\right.$ $v \in \mathscr{K} F,\|v\| \leqslant 1\}$. Далее, (iv) остается в силе после замены равенства (2) на

$$
\|u\|:=\sup \left\{\left\|\mathscr{R}_{w}(u, v)\right\|: v \in \mathscr{K} E,\|v\| \leqslant 1\right\}
$$

$u(6) н a$

$$
\|u\|=\left\|^{\prime} \widetilde{\mathscr{R}}^{u}\right\|_{c b}=\left\|^{\prime} \mathscr{R}_{w}^{u}\right\| .
$$

Пункт (v) принимает вид “норма вектора $x \in \square E$ есть $\left\|^{\prime} \mathscr{R}^{x}\right\|_{c b}$ ", u, наконеи, (vi) остается в силе после замены $E$ на $F$ и F на $E$.

ДокАзАтельство. Сперва покажем, что для любого $v \in \mathscr{K} F$ оператор $\widetilde{\mathscr{R}}^{v}$ полностью ограничен, а для этого по линейности достаточно показать, что таков $\widetilde{\mathscr{R}}^{\text {by }}$ для всех by $\in \mathscr{K} F$. Объединяя первое из равенств (4) с предложением 6 , а также с условиями теоремы, мы получаем, что $\left\|\widetilde{\mathscr{R}}_{\infty}^{b y}(u)\right\|=\left\|b\left[\mathscr{R}_{\infty}^{y}(u)\right]\right\| \leqslant$ $\|b\|\left\|\mathscr{R}^{y}\right\|_{c b}\|u\|$. Отсюда следует (i).

Далее, первое из равенств (5) вместе с определением вторичного квантования влечет $\left\|\mathscr{R}_{w}^{v}(u)\right\|=\left\|\widetilde{\mathscr{R}}_{\infty}^{v}(u)\right\|$ для всех $u \in \mathscr{K} E$. Поэтому из (i) следует (ii) и, вдобавок, равенство $\left\|\mathscr{R}_{w}^{v}\right\|=\left\|\widetilde{\mathscr{R}}^{v}\right\|_{c b}$. В свою очередь, из (ii) очевидным образом следует (iii) вместе с равенством $\left\|\mathscr{R}_{w}^{v}\right\|=\sup \left\{\left\|\mathscr{R}_{w}(u, v)\right\|: u \in \mathscr{K} E\right.$, $\|u\| \leqslant 1\}$.

Итак, число $\|v\|$ корректно определено и, в частности, оно является нормой оператора $\mathscr{R}_{w}^{\mathscr{K} F}(v)$. Отсюда с учетом линейности отображения $\mathscr{R}_{w}^{\mathscr{K} F}$ следует, что функция $v \mapsto\|v\|$ является преднормой в $\mathscr{K} F$.

Покажем, что это норма. Пусть $v \neq 0$. Тогда, как хорошо известно (см., например, [13]), $v$ имеет вид $\sum_{k=1}^{n} b_{k} y_{k}$, где система $b_{k}$ линейно независима, а $y_{1} \neq 0$. Согласно условию существует $x \in E$ такой, что $\mathscr{R}\left(x, y_{1}\right) \neq 0$. Возьмем любой $a \in \mathscr{K}, a \neq 0$. Тогда оператор $\mathscr{R}_{w}^{\mathscr{K} F}(v) \in \mathscr{B}(\mathscr{K} E, \mathscr{K} G)$ переводит ах в $\mathscr{R}_{w}(a x, v)=\sum_{k=1}^{n} \mathscr{R}_{w}\left(a x, b_{k} y_{k}\right)=\sum_{k=1}^{n}\left(a \diamond b_{k}\right) \mathscr{R}\left(x, y_{k}\right)$. Но система операторов $a \diamond b_{k}$ очевидным образом линейно независима вместе с системой $b_{k}$, а $\mathscr{R}\left(x, y_{1}\right) \neq 0$. Поэтому (см. там же) $\mathscr{R}_{w}(a x, v) \neq 0$, а значит, и $\mathscr{R}_{w}^{\mathscr{K} F}(v) \neq 0$. Отсюда, разумеется, вытекает, что $\|v\| \neq 0$.

Теперь напомним, что $\mathscr{B}$-бимодуль $\mathscr{K} G$, обладая аксиомами Руана относительно исходных внешних умножений “.", обладает теми же аксиомами и относительно внешних умножений " .2" (предложение 2). Отсюда в силу предложения 3 теми же аксиомами обладает и $\mathscr{B}$-бимодуль $\mathscr{B}(\mathscr{K} E, \mathscr{K} G)$ относительно соответствующих индуцированных операций. Далее, имеют место равенства $\mathscr{R}_{w}(u, c \cdot v)=c \cdot_{2} \mathscr{R}_{w}(u, v)$ и $\mathscr{R}_{w}(u, v \cdot c)=\mathscr{R}_{w}(u, v) \cdot_{2} c, c \in \mathscr{B}$, очевидным образом проверяемые на элементарных тензорах в $\mathscr{K} F$ и $\mathscr{K} E$. Из них немедленно следует, что оператор $\mathscr{R}_{w}^{\mathscr{K} F}: \mathscr{K} F \rightarrow \mathscr{B}(\mathscr{K} E, \mathscr{K} G)$ является морфизмом 
$\mathscr{B}$-бимодулей относительно стандартных операций в $\mathscr{K} F$ и только что упомянутых операций в $\mathscr{B}(\mathscr{K} E, \mathscr{K} G)$. Отсюда согласно предложению 4 вытекает, что построенная норма в $\mathscr{K} F$ является квантовой нормой в $F$. Отсюда следует (iv).

Возьмем $y \in F$ и одномерный проектор $p \in \mathscr{K}$. Тогда $\|p y\|=\left\|\widetilde{\mathscr{R}}^{p y}\right\|_{c b}=$ $\left\|\widetilde{\mathscr{R}}_{\infty}^{p y}\right\|$. В силу первого из равенств (4) и предложения 7 для любого $u \in \mathscr{K} E$ норма $\widetilde{\mathscr{R}}_{\infty}^{p y}(u)$ есть норма элементарного тензора $p\left[\mathscr{R}_{\infty}^{y}(u)\right] \in \mathscr{K}[\mathscr{K} G]$, где, как мы помним, $\mathscr{R}_{\infty}^{y}(u) \in \mathscr{K} G$. Поскольку норма в $\mathscr{K}[\mathscr{K} G]-$ это квантование нормы в $\mathscr{K} G$, мы получаем, что $\|p y\|=\left\|\mathscr{R}_{\infty}^{y}\right\|$. В силу определения нормы в $\square F$ отсюда следует (v).

Наконец, из определения квантовой нормы в $\mathscr{K} F$ с помощью формулы (2) немедленно следует (vi).

Этим завершено доказательство части теоремы, касающейся построения квантовой нормы во втором аргументе биоператора $\mathscr{R}$. Ее часть, касающаяся первого аргумента, доказывается таким же, с точностью до очевидных модификаций, рассуждением. (Можно также, если угодно, применить уже установленную "половину" к биоператору $\mathscr{R}^{o p}: F \times E:(y, x) \mapsto \mathscr{R}(x, y)$.)

Отметим, что в условиях теоремы имеет место коммутативная диаграмма

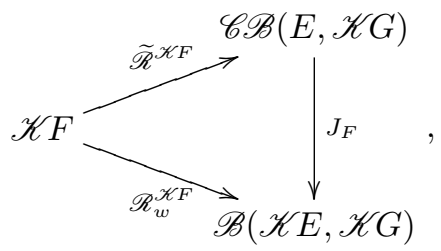

в которой теми же символами $\widetilde{\mathscr{R}}^{\mathscr{K} F}$ и $\mathscr{R}_{w}^{\mathscr{K} F}$ обозначены коограничения введенных выше операторов, а $J_{F}$ - изометрический оператор, переводящий $\varphi: E \rightarrow$ $\mathscr{K} G$ в $\psi: \mathscr{K} E \rightarrow \mathscr{K} G: u \mapsto \varsigma_{G} \varphi_{\infty}(u)$. Действительно, из определения вторичного квантования пространства $G$ следует, что для $\varphi \in \mathscr{C} \mathscr{B}(E, \mathscr{K} G)$ и $u \in \mathscr{K} E$ выполнено $\left\|\left[J_{F} \varphi\right] u\right\|=\left\|\varphi_{\infty}(u)\right\|$. Отсюда сразу вытекает, что $J_{F}$ корректно определен и является изометрическим оператором. Коммутативность диаграммы обеспечена первым из равенств (5).

\section{§ 3. Квантование пространств операторов, квантовый экспоненциальный закон и квантовая сопряженная ассоциативность}

Особенно важны два специальных случая описанного квантования: когда $G=\mathbb{C}$ и когда $F=\mathscr{C} \mathscr{B}(E, G)$. Первому из них мы посвятим следующий параграф, а пока займемся вторым.

Пусть $E$ и $G$ - квантовые пространства. Рассмотрим "биоператор означивания" $\mathscr{E}: E \times \mathscr{C} \mathscr{B}(E, G) \rightarrow G:(x, \varphi) \mapsto \varphi(x)$ и его слабое размножение $\mathscr{E}_{w}: \mathscr{K} E \times \mathscr{K}[\mathscr{C} \mathscr{B}(E, G)] \rightarrow \mathscr{K} G$, которое в данной ситуации, очевидно, однозначно определено правилом $(a x, b \varphi) \mapsto(a \diamond b) \varphi(x)$. Ясно, что при $F:=$ $\mathscr{C} \mathscr{B}(E, G)$ и $\mathscr{R}:=\mathscr{E}$ условия теоремы 1 (они же и условия первой "половины" теоремы $\left.1^{\prime}\right)$ выполнены. 
При этом для $\Phi \in \mathscr{K}[\mathscr{C} \mathscr{B}(E, G)]$ операторы $\mathscr{R}_{w}^{v}$ приобретают вид $\mathscr{E}_{w}^{\Phi}: \mathscr{K} E \rightarrow$ $\mathscr{K} G: u \mapsto \mathscr{E}_{w}(u, \Phi)$, а операторы $\widetilde{\mathscr{R}}^{v}-$ вид $\widetilde{\mathscr{E}}^{\Phi}: E \rightarrow \mathscr{K} G: x \mapsto^{\prime} \mathscr{E}_{\infty}(\Phi)$, где $\mathscr{E}^{\mathscr{E}}: \varphi \mapsto \varphi(x)$; в частности, $\widetilde{\mathscr{R}}^{b \varphi}: E \rightarrow \mathscr{K} G$ переводит $x$ в $b \varphi(x)$.

Ясно, что п. (v) теоремы $1^{\prime}$ применительно к $\mathscr{C} \mathscr{B}(E, G)$ означает, что полученное квантовое пространство есть квантование нормированного пространства $\left(\mathscr{C} \mathscr{B}(E, G),\|\cdot\|_{c b}\right)$. Это квантование, а также соответствующую квантовую норму мы будем называть стандартными. Таким образом, для $\Phi \in$ $\mathscr{K}[\mathscr{C} \mathscr{B}(E, G)]$ выполнено

$$
\|\Phi\|:=\sup \left\{\left\|\mathscr{E}_{w}(u, \Phi)\right\|: u \in \mathscr{K} E,\|u\| \leqslant 1\right\}
$$

и, кроме того,

$$
\|\Phi\|:=\|\widetilde{\mathscr{E}} \Phi\|_{c b}=\left\|\mathscr{E}_{w} \Phi\right\|
$$

(первое из равенств (8) по существу содержится у Пизье; ср. его "вложение $\mathscr{K} \otimes_{\alpha} C B(E, F) \subset C B\left(E, \mathscr{K} \otimes_{\min } F\right) "$ в [5; с. 41]).

Примем краткие обозначения $\alpha_{E, G}$ и $\beta_{E, G}$ для операторов $\mathscr{K}[\mathscr{C} \mathscr{B}(E, G)] \rightarrow$ $\mathscr{C} \mathscr{B}(E, \mathscr{K} G): \Phi \mapsto \widetilde{\mathscr{E}}^{\Phi}$ и $\mathscr{K}[\mathscr{C} \mathscr{B}(E, G)] \rightarrow \mathscr{B}(\mathscr{K} E, \mathscr{K} G): \Phi \mapsto \mathscr{E}_{w}^{\Phi}$. Равенства (7)-(8) означают, что это изометрические изоморфизмы. Оба оператора, разумеется, являются специализациями для случая $\mathscr{R}:=\mathscr{E}$ операторов $\widetilde{\mathscr{R}}^{\mathscr{K}}$ и и $\mathscr{R}_{w}^{\mathscr{K} F}$, фигурирующих в теореме $1^{\prime}$. В частности, $\beta_{E, G}=J_{\mathscr{C} \mathscr{B}(E, G)} \alpha_{E, G}$ (cp. диаграмму (D1)).

Указанная конструкция квантования позволяет говорить о “функторах полностью ограниченных операторов", определенных на категории квантовых пространств (с полностью ограниченными операторами в качестве морфизмов) и принимающих значения в той же категории. Мы не будем в этой статье останавливаться на деталях соответствующих определений, а только приведем утверждение, лежащее в их основе.

ПреДЛОЖЕНИЕ 9. Пусть $E, F u G$ - квантовые пространства, $\varphi: F \rightarrow G-$ полностью ограниченный оператор. Тогда отображения

$$
\begin{aligned}
& \mathscr{C} \mathscr{B}(E, \varphi): \mathscr{C} \mathscr{B}(E, F) \rightarrow \mathscr{C} \mathscr{B}(E, G): \psi \mapsto \varphi \psi, \\
& \mathscr{C} \mathscr{B}(\varphi, E): \mathscr{C} \mathscr{B}(G, E) \rightarrow \mathscr{C} \mathscr{B}(F, E): \psi \mapsto \psi \varphi
\end{aligned}
$$

сами суть полностью ограниченные относительно стандартного квантования операторы. Если же, вдобавок, $\varphi$ - полностью изометрический (соответственно полностъю коизометрический) оператор, то таков же $\mathscr{C} \mathscr{B}(E, \varphi)$ (соответственно $\mathscr{C} \mathscr{B}(\varphi, E))$.

ДокАЗАТЕЛЬСтво. Ясно, что $(\varphi \psi)_{\infty}=\varphi_{\infty} \psi_{\infty}$, и как следствие композиция двух полностью ограниченных операторов сама есть полностью ограниченный оператор. Поэтому указанные отображения корректно определены и, разумеется, линейны. Это позволяет говорить об их размножениях. Рассмотрим диаграмму

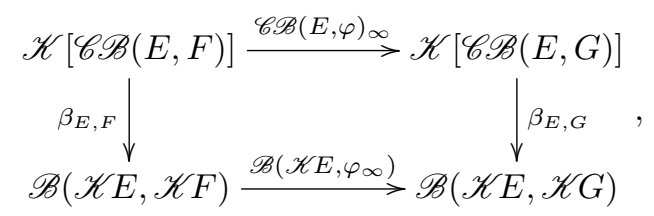


в которой, помимо уже известных нам операторов, $\mathscr{B}\left(\mathscr{K} E, \varphi_{\infty}\right)$ - это ограниченный оператор, получающийся как результат применения к $\varphi_{\infty}$ "классического" функтора операторов $\mathscr{B}(\mathscr{K} E, \cdot)$ (см., например, [13; предложение 2.5.1]).

Простая проверка на элементарных тензорах показывает, что наша диаграмма коммутативна. Но ее вертикальные стрелки изображают изометрические операторы. Поэтому поскольку $\mathscr{B}\left(\mathscr{K} E, \varphi_{\infty}\right)$ ограничен (см. там же), то таков же и $\mathscr{C} \mathscr{B}(E, \varphi)_{\infty}$. Если же, вдобавок, $\varphi_{\infty}$ - изометрический оператор, то, очевидно, этим же свойством обладает и $\mathscr{B}\left(\mathscr{K} E, \varphi_{\infty}\right)$, а значит, таков же и $\mathscr{C} \mathscr{B}(E, \varphi)_{\infty}$

Этим доказаны утверждения, касающиеся $\mathscr{C} \mathscr{B}(E, \varphi)$; аналогичным рассуждением устанавливаются утверждения о $\mathscr{C} \mathscr{B}(\varphi, E)$.

В качестве приложения отметим полезное

ПРеДЛОЖЕНИЕ 10. Пусть Е $и G$ - квантовые пространства. Тогда $\alpha_{E, G}-$ (не только изометрический, но и) полностью изометрический оператор.

ДоказАтельство. Рассмотрим диаграмму

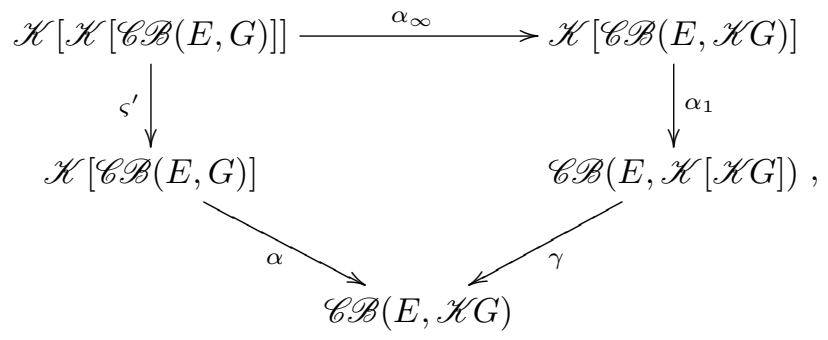

в которой $\alpha:=\alpha_{E, G}, \alpha_{1}:=\alpha_{E, \mathscr{K} G}, \varsigma^{\prime}:=\varsigma_{\mathscr{C} \mathscr{B}(E, G)}, \gamma:=\mathscr{C} \mathscr{B}\left(E, \varsigma_{G}\right)$ и $\alpha_{\infty}:=$ $\left(\alpha_{E, G}\right)_{\infty}$. Она, как легко проверить на элементарных тензорах, коммутативна. Но нам уже известно, что из перечисленных операторов три первых - изометрические. Что же касается $\gamma$, то, объединяя предложения 8 и 9, мы видим, что это тоже изометрический (и даже полностью изометрический) оператор. Значит, изометрическим является и $\alpha_{\infty}$. Дальше ясно.

Теперь напомним об общематематическом “экспоненциальном законе"

$$
\left(X^{Y}\right)^{Z}=X^{Y \times Z}=\left(X^{Z}\right)^{Y}
$$

$(X, Y, Z$ - множества), связывающем функции двух переменных с функциями одного переменного со значениями в множестве функций от другого переменного. Как хорошо известно, в классическом функциональном анализе этот закон принимает следующую специальную форму. Пусть $E, F, G$ - нормированные пространства, $\mathscr{B}(E \times F, G)$ - пространство всех ограниченных биоператоров uз $E \times F$ в $G$, снабженное биоператорной нормой. Тогда корректно определена диаграмма

$$
\mathscr{B}(F, \mathscr{B}(E, G)) \stackrel{I_{F}}{\longleftarrow} \mathscr{B}(E \times F, G) \stackrel{{ }^{\prime} I_{E}}{\longrightarrow} \mathscr{B}(E, \mathscr{B}(F, G))
$$


где $I_{F}$ и' $I_{E}$ - изометрические изоморфизмы нормированных пространств, переводящие (в обозначениях предыдущего параграфа) биоператор $\mathscr{R}$ в оператоpы соответственно $\mathscr{R}^{F}$ u ' $\mathscr{R}^{E}$. Наша очередная цель-рассмотреть квантовую версию этого экспоненциального закона.

Ранее был введен оператор означивания $\mathscr{E}$, заданный на $E \times \mathscr{C} \mathscr{B}(E, G)$. Обозначим тем же символом $\mathscr{E}$ его продолжение на $E \times \mathscr{B}(E, G)$, действующее по тому же правилу $(x, \varphi) \mapsto \varphi(x)$; это не вызовет путаницы. В дальнейших рассуждениях нам пригодится формула

$$
\mathscr{E}_{w}\left(u, \mathscr{R}_{\infty}^{F}(v)\right)=\mathscr{R}_{w}(u, v),
$$

имеющая место для всех $\mathscr{R} \in \mathscr{B}(E \times F, G), u \in \mathscr{K} E, v \in \mathscr{K} F$. Действительно, для элементарных тензоров в соответствующих пространствах выполнено

$$
\begin{aligned}
\mathscr{E}_{w}\left(a x, \mathscr{R}_{\infty}^{F}(b y)\right) & =\mathscr{E}_{w}\left(a x, b\left[\mathscr{R}^{F}(y)\right]\right)=\mathscr{E}_{w}\left(a x, b \mathscr{R}^{y}\right) \\
& =(a \diamond b) \mathscr{E}\left(x, \mathscr{R}^{y}\right)=(a \diamond b) \mathscr{R}(x, y)=\mathscr{R}_{w}(a x, b y),
\end{aligned}
$$

и общий случай немедленно следует из (би)линейности рассматриваемых отображений.

Итак, пусть $E, F$ и $G$ - квантовые пространства.

ТЕОРема 2. Существуют изометрические изоморфизмы (нормированных пространств) $I_{F} u^{\prime} I_{E}$, участвующие в корректно определенной диаграмме

$$
\mathscr{C} \mathscr{B}(F, \mathscr{C} \mathscr{B}(E, G)) \stackrel{I_{F}}{\longleftarrow} \mathscr{W} \mathscr{B}(E \times F, G) \stackrel{{ }^{\prime} I_{E}}{\longrightarrow} \mathscr{C} \mathscr{B}(E, \mathscr{C} \mathscr{B}(F, G))
$$

и действующие по тем же правилам, что и операторы диаграммы (D2).

Более подробно:

(i) для любого $\mathscr{R} \in \mathscr{W} \mathscr{B}(E \times F, G)$ u $y \in F$ оператор $\mathscr{R}^{y}: E \rightarrow G$ полностью ограничен;

(ii) оператор $\mathscr{R}^{F}: F \rightarrow \mathscr{C} \mathscr{B}(E, G): y \mapsto \mathscr{R}^{y}$, корректно определенный в силу (i), полностъю ограничен относительно стандартной квантовой норми в $\mathscr{C} \mathscr{B}(E, G)$;

(iii) оператор $I_{F}: \mathscr{W} \mathscr{B}(E \times F, G) \rightarrow \mathscr{C} \mathscr{B}(F, \mathscr{C} \mathscr{B}(E, G))$, корректно определенный в силу (ii), является изометрическим изоморфизмом.

Аналогичное утверждение об операторах ${ }^{1} \mathscr{R}^{x}, \mathscr{R}^{F}$ u $I_{E}$ также имеет место.

ДокАЗАТЕЛЬСтво. Возьмем одномерный проектор $p \in \mathscr{K}, y \in F$ и $u \in \mathscr{K} E$. Объединяя первые равенства из (4) и (5), мы видим, что

$$
\varsigma_{G} \mho_{G}\left(p\left[\mathscr{R}_{\infty}^{y}(u)\right]\right)=\varsigma_{G} \widetilde{\mathscr{R}}_{\infty}^{p y}(u)=\mathscr{R}_{w}^{p y}(u)=\mathscr{R}_{w}(u, p y) .
$$

Поэтому определение вторичного квантования в терминах $\varsigma_{G}$ вместе с предложением 7 дает

$$
\left\|\mathscr{R}_{\infty}^{y}(u)\right\|=\left\|p \mathscr{R}_{\infty}^{y}(u)\right\|=\left\|\mathscr{R}_{w}(u, p y)\right\| \leqslant\|\mathscr{R}\|_{w c b}\|u\|\|p y\|=\|\mathscr{R}\|_{w c b}\|y\|\|u\| .
$$

Отсюда следует (i). Теперь мы вправе говорить об операторе $\mathscr{R}^{F}$ из п. (ii) и его размножении $\mathscr{R}_{\infty}^{F}: \mathscr{K} F \rightarrow \mathscr{K}[\mathscr{C} \mathscr{B}(E, G)]$. Согласно равенству (6) для $v \in \mathscr{K} F$ 
выполнено $\left\|\mathscr{R}_{\infty}^{F}(v)\right\|=\sup \left\{\left\|\mathscr{E}_{w}\left(u, \mathscr{R}_{\infty}^{F}(v)\right)\right\|: u \in \mathscr{K} E,\|u\| \leqslant 1\right\}$. Пользуясь формулой $(9)$, мы видим, что $\left\|\mathscr{R}_{\infty}^{F}(v)\right\|=\sup \left\{\left\|\mathscr{R}_{w}(u, v)\right\|: u \in \mathscr{K} E,\|u\| \leqslant 1\right\}$. Отсюда $\mathscr{R}_{\infty}^{F}$ - ограниченный оператор с нормой, равной $\left\|\mathscr{R}_{w}\right\|$. Это, разумеется, дает (ii), а также равенство $\left\|\mathscr{R}^{F}\right\|_{c b}=\|\mathscr{R}\|_{w c b}$.

Итак, $I_{F}: \mathscr{W} \mathscr{B}(E \times F, G) \rightarrow \mathscr{C} \mathscr{B}(F, \mathscr{C} \mathscr{B}(E, G))$ корректно определен и к тому же является изометрическим оператором. Чтобы завершить доказательство п. (iii), мы покажем, что этот оператор сюръективен.

Возьмем $\mathscr{S} \in \mathscr{C} \mathscr{B}(F, \mathscr{C} \mathscr{B}(E, G))$ и положим $\mathscr{R}: E \times F \rightarrow G:(x, y) \mapsto[\mathscr{S}(y)](x)$.

Очевидно, $\mathscr{R}$ ограничен и $\mathscr{R}^{F}=\mathscr{S}$. Поэтому наша задача - убедиться в том, что $\mathscr{R}$ слабо полностью ограничен. Но из той же формулы (9) следует, что $\left\|\mathscr{R}_{w}(u, v)\right\|=\left\|\mathscr{E}_{w}\left(u, \mathscr{S}_{\infty}(v)\right)\right\|$. Далее, мы знаем, что $\|\mathscr{E}\|_{w c b}=1$ : это специальный случай теоремы $1^{\prime},(\mathrm{vi})$, когда исходный биоператор задан как биоператор означивания. Отсюда $\left\|\mathscr{R}_{w}(u, v)\right\| \leqslant\|u\|\left\|\mathscr{S}_{\infty}(v)\right\| \leqslant\|\mathscr{S}\|_{\infty}\|u\|\|v\|$. Тем самым, $\mathscr{R} \in \mathscr{W} \mathscr{B}(E \times F, G)$.

Часть теоремы, относящаяся к оператору $I_{F}$, полностью доказана. "Симметричное" утверждение об $I_{E}$ доказывается аналогичным рассуждением.

Теперь напомним о существовании “четырехименного" тензорного произведения квантовых пространств, осуществляющего линеаризацию слабо полностью ограниченных биоператоров (см. конец §1). Следующее утверждение представляет собой квантовую версию так называемого "закона сопряженной ассоциативности" классического функционального анализа (см., например, [13; $\S 2.7])$.

Теорема 3. Для тех же $E, F, G$ существуют полностью изометрические

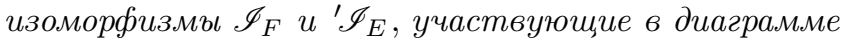

$$
\mathscr{C} \mathscr{B}(F, \mathscr{C} \mathscr{B}(E, G)) \stackrel{\mathscr{I}_{F}}{\longleftarrow} \mathscr{C} \mathscr{B}\left(E \otimes_{4} F, G\right) \stackrel{\mathscr{I}_{E}}{\longrightarrow} \mathscr{C} \mathscr{B}(E, \mathscr{C} \mathscr{B}(F, G))
$$

и однозначно определенные равенствами

$$
\left(\left[\mathscr{I}_{F}(\varphi)\right] y\right)(x)=\varphi(x \otimes y)=\left(\left[\mathscr{I}_{E}(\varphi)\right] x\right)(y)
$$

ДокАзАтельство. Обозначим через $i: \mathscr{W} \mathscr{B}(E \times F, G) \rightarrow \mathscr{C} \mathscr{B}\left(E \otimes_{4} F, G\right)$ отображение, сопоставляющее биоператору его линеаризацию (ассоциированный линейный оператор); как упоминалось в конце $§ 1$, это изометрический изоморфизм нормированных пространств. Положим $\mathscr{I}_{F}:=I_{F} i^{-1}$. Тогда из предыдущей теоремы очевидным образом следует, что это изометрический (пока просто изометрический) изоморфизм, однозначно определенный на элементарных тензорах в $E \otimes F$ первым из указанных выше равенств. Это, разумеется, верно для всех троек квантовых пространств. Поэтому оператор $\mathscr{I}_{F, \mathscr{K} G}$, определяемый дословно как $\mathscr{I}_{F}$, но с $\mathscr{K} G$ в роли $G$, - также (изометрический) изоморфизм. 
Теперь рассмотрим диаграмму

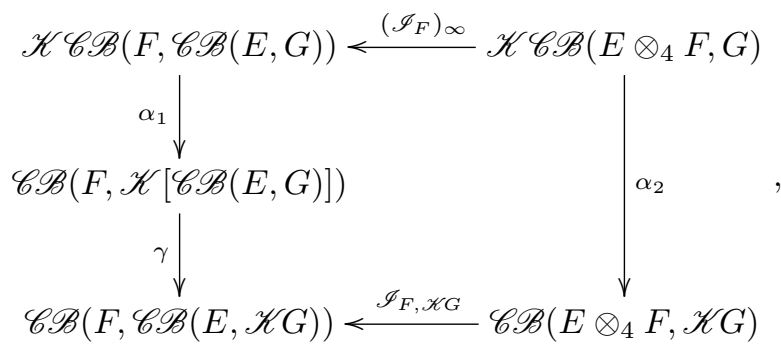

в которой $\alpha_{1}:=\alpha_{F, \mathscr{C} \mathscr{B}(E, G)}, \alpha_{2}:=\alpha_{E \otimes_{4} F, G}$, а $\gamma=\mathscr{C} \mathscr{B}\left(F, \alpha_{E, G}\right)$ (см. предложение 9). На основании предложения 10 все операторы $\alpha_{(\cdot, \cdot)}$ являются полностью изометрическими. Отсюда в силу предложения 9 следует, что таков же и $\gamma$. Наконец, как уже отмечалось, $\mathscr{I}_{F, \mathscr{K} G}$ - также изометрический оператор. Но наша диаграмма, очевидно, коммутативна. Отсюда сразу следует, что и $\left(\mathscr{I}_{F}\right)_{\infty}$ - изометрический оператор, а значит, поскольку это биекция, изометрический изоморфизм.

Итак, $\mathscr{I}_{F}-$ полностью изометрический изоморфизм; то, что таков же и ' $\mathscr{I}_{E}$, доказывается аналогичным рассуждением.

ЗАмЕчАнИЕ 4. Как и в случае сопряженной ассоциативности классического функционального анализа, за доказанной теоремой скрывается естественная эквивалентность возникающих трехместных функторов, которые переводят тройку объектов $(E, F, G)$ соответственно в $\mathscr{C} \mathscr{B}(F, \mathscr{C} \mathscr{B}(E, G)), \mathscr{C} \mathscr{B}\left(E \otimes_{4} F, G\right)$ и $\mathscr{C} \mathscr{B}(E, \mathscr{C} \mathscr{B}(F, G))$.

\section{§ 4. Квантовое сопряженное и квантовое предсопряженное пространства. Пример: квантовое пространство ядерных операторов}

В этом параграфе мы сосредоточимся на случае, когда задана обычная (т.е. не векторная, а скалярная) двойственность $\mathscr{D}: E \times F \rightarrow \mathbb{C}$. В силу естественного отождествления $\mathscr{K} \mathbb{C}$ с $\mathscr{K}$ соответствующее слабое размножение может быть рассмотрено как биоператор $\mathscr{D}_{w}: \mathscr{K} E \times \mathscr{K} F \rightarrow \mathscr{K}$, однозначно определенный правилом $(a x, b y) \mapsto \mathscr{D}(x, y) a \diamond b$; отсюда, в частности, следует, что $\mathscr{D}_{w}^{\mathscr{K} F}: \mathscr{K} F \rightarrow \mathscr{L}(\mathscr{K} E, \mathscr{K})$ переводит by в оператор $a x \mapsto \mathscr{D}(x, y) a \diamond b$. Что же касается $\widetilde{D}^{\mathscr{K} F}: \mathscr{K} F \rightarrow \mathscr{L}(E, \mathscr{K})$, то он переводит by в оператор $x \mapsto \mathscr{D}(x, y) b$; обратим внимание на то, что этот оператор одномерен.

Принципиально важный для дальнейшего факт состоит в том, что для функционалов обычная ограниченность влечет полную.

Если $H$ - гильбертово пространство, а $\xi, \eta$ - его векторы, то через $\xi \circ \eta$ : $H \rightarrow H$ мы обозначим одномерный оператор, переводящий $\zeta$ в $\langle\zeta, \eta\rangle \xi$. Выделим очевидные равенства

$$
(\xi \circ \eta)\left(\xi^{\prime} \circ \eta^{\prime}\right)=\left\langle\xi^{\prime}, \eta\right\rangle\left(\xi \circ \eta^{\prime}\right) \quad \text { и } \quad a(\xi \circ \eta)=a(\xi) \circ \eta,
$$

где $\xi, \xi^{\prime}, \eta, \eta^{\prime} \in H, a \in \mathscr{B}(H)$. 
ПРеДЛОЖЕНИЕ 11. Пусть $f: E \rightarrow \mathbb{C}$ - ограниченный функционал, заданнъй на квантовом пространстве. Тогда $f$ (автоматически) полностъю ограничен $u\|f\|_{c b}=\|f\|$.

ДоказАтельство. Рассмотрим $f_{\infty}: \mathscr{K} E \rightarrow \mathscr{K} \mathbb{C}=\mathscr{K}$ и возьмем $u \in \mathscr{K} E$. В силу свойств операторной нормы

$$
\left\|f_{\infty}(u)\right\|=\sup \left\{\left|\left\langle f_{\infty}(u) \xi, \eta\right\rangle\right|, \xi, \eta \in L,\|\xi\|,\|\eta\| \leqslant 1\right\} .
$$

Произвольно выберем вектор $e \in L,\|e\|=1$, и возьмем проектор $p=e$ о $е$ на его линейную оболочку. Используя формулы (10), а также то, что $f_{\infty}-$ морфизм $\mathscr{B}$-бимодулей (см. §1), мы видим, что

$$
\begin{aligned}
\left\langle\left[f_{\infty}(u)\right](\xi), \eta\right\rangle p & =\left\langle\left[f_{\infty}(u)\right](\xi), \eta\right\rangle(e \circ e)=(e \circ \eta)\left(\left[f_{\infty}(u)\right](\xi) \circ e\right) \\
& =(e \circ \eta) f_{\infty}(u)(\xi \circ e)=f_{\infty}[(e \circ \eta) \cdot u \cdot(\xi \circ e)] .
\end{aligned}
$$

Поэтому $\left|\left\langle\left[f_{\infty}(u)\right](\xi), \eta\right\rangle\right|=\left\|f_{\infty}[(e \circ \eta) \cdot u \cdot(\xi \circ e)]\right\|$.

Теперь заметим, что $(e \circ \eta) \cdot u \cdot(\xi \circ e)$ - это элементарный тензор вида $p x_{\xi, \eta}$ для некоторого $x_{\xi, \eta} \in E$. (Такое равенство очевидно, когда $u$ - сам элементарный тензор, а значит, верно и для всех $u$.) При этом из аксиомы (RI), а также известных (и очевидных) равенств $\|e \circ \eta\|=\|e\|\|\eta\|,\|\xi \circ e\|=\|e\|\|\xi\|$ следует, что при $\|\xi\|,\|\eta\| \leqslant 1$ выполнено $\left\|x_{\xi, \eta}\right\| \leqslant\|u\|$. Поэтому при тех же $\xi, \eta$

$$
\left|\left\langle\left[f_{\infty}(u)\right](\xi), \eta\right\rangle\right|=\left\|f_{\infty}\left(p x_{\xi, \eta}\right)\right\|=\left\|f\left(x_{\xi, \eta}\right) p\right\|=\left|f\left(x_{\xi, \eta}\right)\right| \leqslant\|f\|\left\|x_{\xi, \eta}\right\| \leqslant\|f\|\|u\| .
$$

Переходя к соответствующей верхней грани, мы видим, что $\left\|f_{\infty}\right\| \leqslant\|f\|$. Дальше ясно.

В свете сказанного теорема $1^{\prime}$ при $G:=\mathbb{C}$ приобретает несколько более прозрачный вид, который мы сейчас приведем. Везде далее пространство $\mathscr{K}=\mathscr{K} \mathbb{C}$ рассмотрено с конкретным квантованием, или, что то же самое, со вторичным квантованием комплексной плоскости; см. §1.

ТЕОРема $1^{\prime \prime}$. Пусть $E$ - квантовое пространство, $F$ - линейное пространство, а $\mathscr{D}: E \times F \rightarrow \mathbb{C}-$ двойственность. Пусть, далее, для любого у $\in F$ функиионал $\mathscr{D}^{y}: E \rightarrow \mathbb{C}: x \mapsto \mathscr{D}(x, y)$ ограничен. Тогда $F$ обладает квантовой нормой, корректно определенной равенством

$$
\|v\|:=\sup \left\{\left\|\mathscr{D}_{w}(u, v)\right\|: u \in \mathscr{K} E,\|u\| \leqslant 1\right\},
$$

и для этой же квантовой нормы имеют смысл и справедливы равенства

$$
\|v\|=\left\|\widetilde{\mathscr{D}}^{v}\right\|_{c b}=\left\|\mathscr{D}_{w}^{v}\right\|
$$

При этом (обычная) норма вектора $y \in \square F$ есть $\left\|\mathscr{D}^{y}\right\|$. Наконеи, бибункиионал $\mathscr{D}$ слабо полностъю ограничен относительно исходной квантовой нормы в $E$ и построенной квантовой нормы в $F$; более того, $\|\mathscr{D}\|_{w c b}=1$.

“Симметричная часть". Если $E, F, G$ и $\mathscr{D}$ таковы, что для любого $x \in E$

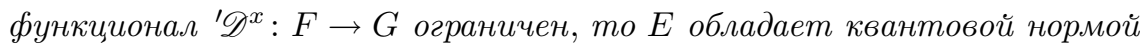

$$
\|u\|:=\sup \left\{\left\|\mathscr{D}_{w}(u, v)\right\|: v \in \mathscr{K} F,\|v\| \leqslant 1\right\},
$$


причем $\|u\|=\left\|^{\prime} \widetilde{\mathscr{D}}^{u}\right\|_{c b}=\left\|^{\prime} \mathscr{D}_{w}^{u}\right\|$, и для $x \in \square E$ выполнено $\|x\|=\left\|^{\prime} \mathscr{D}^{x}\right\|$. Далее, относительно исходной квантовой нормы в $F$ и построенной квантовой нормы в Е выполнено $\|' \mathscr{D}\|_{w c b}=1$.

ДокАЗАТЕЛЬСтво. Предыдущее предложение гарантирует выполнение условий теоремы $1^{\prime}$ при $G:=\mathbb{C}$ и $\mathscr{R}:=\mathscr{D}$. Дальше ясно.

О квантовом пространстве $F$ (соответственно $E$ ) из теоремы $1^{\prime \prime}$ мы будем говорить, что оно порождено заданным квантовым пространством $E$ (соответственно $F$ ) и двойственностью $\mathscr{D}$. Если ясно, о каких квантовом пространстве или двойственности идет речь, упоминание о них будет опускаться.

Наиболее важен, конечно, случай, когда наша двойственность - это каноническая двойственность между нормированным пространством $E$ и его сопряженным $E^{*}$. Ясно, что в этом случае выполнены условия теоремы $1^{\prime \prime}$ как в ее первой "половине" - о квантовании второго аргумента, теперь $E^{*}$, так и во второй - о квантовании $E$. (Заметим только, что последнее верно не "задаром", а вследствие изометричности канонического вложения $E$ в $E^{* *}$.)

ОПРеДЕЛЕНИЕ. Пусть $E$ - нормированное пространство, снабженное квантованием. Тогда пространство $E^{*}$, рассмотренное с квантованием, порожденным канонической двойственностью, называется квантовым сопряженным пространством $\propto$ Е.

Аналогичным образом, если квантованием снабжено $E^{*}$, определяется квантовое предсопряженное пространство $к \mathrm{E}^{*}$.

Разумеется, квантование сопряженного пространства - это частный случай стандартного квантования пространства $\mathscr{C} \mathscr{B}(E, G)$ при $G:=\mathbb{C}$.

ЗАмЕчАниЕ 5. "Безматричное" определение квантового сопряженного пространства впервые упомянуто в работе Пизье [5; с. 40]. Оно дано в терминах (с точностью до обозначений) оператора $\widetilde{D}^{\mathscr{K} E^{*}}: \mathscr{K} E^{*} \rightarrow \mathscr{C} \mathscr{B}(E, \mathscr{K})$, совпадающего также с $\alpha_{E, G}$ из $\S 3$ для $G:=\mathbb{C}$. Этот оператор можно считать разновидностью хорошо известного оператора Гротендика (см., например, [13; предложение 2.7.2, теорема 2.7.7]). Образ оператора $\widetilde{\mathscr{D}}^{\mathscr{K} E^{*}}=\alpha_{E, \mathbb{C}}$ состоит, как легко видеть, из всех ограниченных конечномерных операторов между $E$ и $\mathscr{K}$.

Там, где это не вызовет путаницы, мы будем обозначать бифункционал канонической двойственности между пространством и его сопряженным через $\langle\cdot, \cdot\rangle$, а его слабое размножение - через $\langle\langle\cdot, \cdot\rangle\rangle$.

ПредлОЖениЕ 12. Пусть $E^{*}$ снабжено квантованием. Тогда его квантовое предсопряженное пространство Е совпадает с соответствующим квантовым подпространством квантового сопряженного $\kappa E^{*}$ пространства $E^{* *}$. Более точно, каноническое вложение $i: E \rightarrow E^{* *}$ есть полностъю изометрический оператор.

ДоказАтельство. Возьмем $u \in \mathscr{K} E$ и $v \in \mathscr{K} E^{*}$. С учетом формулы (1) из $\S 1$ простая проверка на элементарных тензорах показывает, что $\langle\langle u, v\rangle\rangle=$ $\triangle\left\langle\left\langle v, i_{\infty}(u)\right\rangle\right\rangle$. Отсюда в силу предложения $1\|\langle\langle u, v\rangle\rangle\|=\left\|\left\langle\left\langle v, i_{\infty}(u)\right\rangle\right\rangle\right\|$. Но согласно второй части предыдущего определения

$$
\left\|i_{\infty}(u)\right\|=\sup \left\{\left\|\left\langle\left\langle v, i_{\infty}(u)\right\rangle\right\rangle\right\|: v \in \mathscr{K} E^{*},\|v\| \leqslant 1\right\},
$$


в то время как первая часть дает

$$
\|u\|=\sup \left\{\|\langle\langle u, v\rangle\rangle\|: v \in \mathscr{K} E^{*},\|v\| \leqslant 1\right\} .
$$

Дальше ясно.

ЗАмечание 6 . Если $E$ - квантовое пространство, $E^{*}$ - его квантовое сопряженное, а $E^{* *}$ - его квантовое второе сопряженное (т.е. квантовое сопряженное к $E^{*}$ ), то каноническое вложение $i: E \rightarrow E^{* *}$ также является полностью изометрическим оператором. Однако этот факт гораздо глубже предыдущего предложения, и он опирается на трудно доказываемое равенство $\|u\|=$ $\sup \left\{\left\|\varphi_{\infty}(u)\right\| ; \varphi \in \mathscr{C} \mathscr{B}(E, \mathscr{K}),\|\varphi\|_{c b} \leqslant 1\right\}, u \in E$. (Оно же играет главную роль в доказательстве теоремы Руана, упомянутой в замечании 1.)

Далее, при тех же $E$ и $E^{*}$ мы можем рассмотреть $E$ как квантовое предсопряженное пространство к $E^{*}$. Нетрудно показать, опираясь на только что указанное свойство канонического вложения, что мы получим не что иное, как исходное квантовое пространство. Однако доказательство приведенного выше равенства для $\|u\|$ и его следствий выходит за пределы настоящей статьи.

ЗАмЕчАние 7 . Пусть, напротив, первоначально задано квантование пространства $E^{*}$, в $E$ рассмотрена структура квантового предсопряженного пространства и, наконец, $E^{*}$ рассмотрено как квантовое сопряженное к этому последнему. Получим ли мы исходную структуру квантового пространства в $E^{*}$ ? Оказывается, не всегда (хотя в большинстве известных примеров это действительно так). Более того, как показал Ле Мерди [14] (в рамках "матричного" подхода), некоторые сопряженные банаховы пространства можно проквантовать так, что получившееся квантовое пространство не является квантовым сопряженным ни к какому квантовому пространству.

Чем оборачивается определение квантовой нормы, порожденной двойственностью, при рассмотрении наиболее известных пространств, встречающихся в анализе? В этой статье мы ограничимся лишь одним важным примером. Речь пойдет о пространстве ядерных операторов (называемых также операторами следового класса), действующих в некотором гильбертовом пространстве $H$; обозначим его через $\mathscr{N}(H)$. Оно снабжено так называемой ядерной нормой $\|y\|_{\mathscr{N}}:=\inf \left\{\sum_{n=1}^{\infty}\left\|z_{n}\right\|\right\}$, где нижняя грань взята по всевозможным представлениям $y$ в виде суммы абсолютно сходящегося ряда одномерных операторов $z_{n}$ (см., например, [13; § 2.7]).

Желая проквантовать пространство $\mathscr{N}(H)$, мы рассмотрим наряду с ним пространство $\mathscr{K}(H)$, на этот раз с обычной операторной нормой. Оба пространства связаны двойственностью $\mathscr{T}: \mathscr{K}(H) \times \mathscr{N}(H) \rightarrow \mathbb{C}:(x, y) \mapsto \operatorname{tr}(x y)$, где $\operatorname{tr}(\cdot)$ - операторный след. Как известно, эта двойственность позволяет реализовать $\mathscr{N}(H)$ как сопряженное нормированное пространство к $\mathscr{K}(H)$. Точный смысл сказанного, в обозначениях $\S 2$, состоит в том, что оператор $\mathscr{T}^{\mathscr{N}(H)}: \mathscr{N}(H) \rightarrow \mathscr{K}(H)^{*}$ является изометрическим изоморфизмом. (Это теорема Шэттена-фон Нейманна; см., например, [15; теорема 3.4.4].) Таким образом, для $\mathscr{T}$ и любого квантования пространства $\mathscr{K}(H)$ условия теоремы $1^{\prime \prime}$, касающиеся второго аргумента, выполнены. В частности, в силу этой теоремы возникает квантование пространства $\mathscr{N}(H)$, порожденное нашей двойственностью и конкретным квантованием пространства $\mathscr{K}(H)$. Это квантование мы 
будем называть стандартным. (Его можно рассматривать как специальный случай стандартного квантования пространства $\mathscr{C} \mathscr{B}(E, G)$ при $E=\mathscr{K}(H)$ и $G=\mathbb{C}$.) Подчеркнем, что это квантование не имеет ничего общего с конкретнъмм квантованием $\mathscr{N}(H)$ как (незамкнутого) нормированного подпространства в $\mathscr{B}(H)$.

Введенное квантование позволяет отождествить $\mathscr{N}(H)$ с $\mathscr{K}(H)^{*}$ не только как нормированные, но и как квантовые пространства. Действительно, простая проверка на элементарных тензорах показывает, что для всех $u \in \mathscr{K}[\mathscr{K}(H)]$ и $v \in \mathscr{K}[\mathscr{N}(H)]$ выполнено $\mathscr{T}_{w}(u, v)=\left\langle\left\langle u, \mathscr{T}_{\infty}^{\mathscr{N}(H)}(v)\right\rangle\right.$. Отсюда в силу определений норм элементов $v$ и $\mathscr{T}_{\infty}^{\mathscr{N}(H)}(v) \in \mathscr{K}\left[\mathscr{K}(H)^{*}\right]$ как соответствующей верхней грани мы видим, что $\mathscr{T}_{\infty}^{\mathscr{N}(H)}$ - изометрический оператор. Следовательно, $\mathscr{T}^{\mathscr{N}(H)}$ с учетом его биективности - полностью изометрический изоморфизм.

Поскольку $\left\langle x, \mathscr{T}^{\mathscr{N}(H)}(y)\right\rangle=\mathscr{T}(x, y)={ }^{\prime} \mathscr{T}^{x}(y)$, из теоремы Шэттена-фон Нейманна сразу следует, что условия теоремы $1^{\prime \prime}$, касающиеся первого аргумента, выполнены для $\mathscr{T}$ и любого квантования пространства $\mathscr{N}(H)$. Поэтому мы вправе говорить о квантовой норме в $\mathscr{K}(H)$, составляющей предмет приведенной ниже теоремы.

На протяжении оставшейся части статьи $\{K\}$ - это направленное по включению множество $\{K\}$ всевозможных конечномерных подпространств в $H$, а $P_{K}$ для каждого $K \in\{K\}$ - это действующий в $H$ проектор на $H_{K}$.

Теорема 4. Квантовая норма в $\mathscr{K}(H)$, порожденная двойственностъю $\mathscr{T}$ и стандартной квантовой нормой в $\mathscr{N}(H)$, совпадает с исходной (равносильно, конкретной) квантовой нормой в этом пространстве.

Разумеется, эта теорема представляет собой специальный случай общего факта, упомянутого в замечании 6 (переход от $E$ к $E^{*}$ и “назад"). Но мы ее получим с помощью сравнительно элементарных средств теории операторов.

ДокАЗАТЕЛЬСтво. Конкретную квантовую норму в пространствах $\mathscr{K}(H)$ и $\mathscr{K}=\mathscr{K}(L)$, равно как и стандартную квантовую норму в $\mathscr{N}(H)$, мы будем попрежнему обозначать через $\|\cdot\|$, а рассматриваемую новую квантовую норму в $\mathscr{K}(H)$ - через $\|\cdot\|_{1}$.

Возьмем $u \in \mathscr{K}[\mathscr{K}(H)]$. В силу $\|\mathscr{T}\|_{w c b}=1$ (см. теорему $1^{\prime \prime}$ ) для всех $v \in \mathscr{K}[\mathscr{N}(H)],\|v\| \leqslant 1$, выполнено $\left\|\mathscr{T}_{w}(u, v)\right\| \leqslant\|u\|$. Отсюда, поскольку

$$
\|u\|_{1}=\sup \left\{\left\|\mathscr{T}_{w}(u, v)\right\|: v \in \mathscr{K}[\mathscr{N}(H)],\|v\| \leqslant 1\right\}
$$

(см. "симметричную часть" той же теоремы), мы видим, что $\|u\|_{1} \leqslant\|u\|$. Чтобы установить обратное неравенство, возьмем указанное выше направленное множество $\{K\}$. Как следует из равенства (11), достаточно предъявить направленность $v_{K} \in \mathscr{K}[\mathscr{N}(H)], K \in\{K\}$, такую, что $\left\|v_{K}\right\| \leqslant 1$ для всех $K$ и $\lim _{K}\left\|\mathscr{T}_{w}\left(u, v_{K}\right)\right\|=\|u\|$. Этим мы и займемся.

Для каждого $K \in\{K\}$ зафиксируем произвольный частично изометрический оператор $S_{K}: H \rightarrow L$ с начальным проектором $P_{K}$ и положим

$$
\sigma_{K}: \mathscr{K}(H) \rightarrow \mathscr{K}: x \mapsto S_{K} x S_{K}^{*} .
$$

В каждом $K \in\{K\}$ произвольно выберем ортонормированный базис $g_{1}, \ldots, g_{n}$ и положим для краткости $g_{i j}:=g_{i} \circ g_{j}$. (Этот оператор, будучи одномерным, 
принадлежит обоим пространствам $\mathscr{K}(H)$ и $\mathscr{N}(H)$.) Наконец, в $\mathscr{K}[\mathscr{N}(H)]$ рассмотрим направленность

$$
v_{K}:=\sum_{i, j=1}^{n}\left[\sigma_{K}\left(g_{i j}\right)\right] g_{j i}
$$

Мы видим, что для любых $a \in \mathscr{K}, x \in \mathscr{K}(H)$ выполнено

$$
\mathscr{T}_{w}\left(a x, v_{K}\right)=\sum_{i, j=1}^{n} \operatorname{tr}\left(x g_{i j}\right) a \diamond \sigma_{K}\left(g_{j i}\right)=a \diamond \sigma_{K}\left[\sum_{i, j=1}^{n} \operatorname{tr}\left(x g_{i j}\right)\left(g_{j i}\right)\right] .
$$

Теперь напомним, что для всех $\xi, \eta \in H$ след одномерного оператора $\xi \circ \eta$ есть в точности $\langle\xi, \eta\rangle$. Вместе с формулами (10), касающимися одномерных операторов, это дает

$\operatorname{tr}\left(x g_{i j}\right)\left(g_{j i}\right)=\operatorname{tr}\left(x\left(g_{i}\right) \circ g_{j}\right) g_{j} \circ g_{i}=\left\langle x\left(g_{i}\right), g_{j}\right\rangle g_{j} \circ g_{i}=\left(g_{j} \circ g_{j}\right)\left(x\left(g_{i}\right) \circ g_{i}\right)=g_{j j} x g_{i i}$.

Отсюда для любых $a \in \mathscr{K}, x \in \mathscr{K}(H)$ выполнено

$$
\begin{aligned}
\mathscr{T}_{w}\left(a x, v_{K}\right) & =\sum_{i, j=1}^{n} \operatorname{tr}\left(x g_{i j}\right) a \diamond \sigma_{K}\left(g_{j i}\right)=a \diamond \sigma_{K}\left[\sum_{i, j=1}^{n} \operatorname{tr}\left(x g_{i j}\right)\left(g_{j i}\right)\right] \\
& =a \diamond \sigma_{K}\left[\sum_{i, j=1}^{n} g_{j j} x g_{i i}\right]=a \diamond \sigma_{K}\left[\left(\sum_{i=1}^{n} g_{i i}\right) x\left(\sum_{j=1}^{n} g_{j j}\right)\right] .
\end{aligned}
$$

Ясно, что $\sum_{i=1}^{n} g_{i i}$ - это не что иное, как $P_{K}$. Это означает, что $S_{K} \sum_{i=1}^{n} g_{i i}=S_{K}$ и $\sum_{j=1}^{n} g_{j j} S_{K}^{*}=S_{K}^{*}$. В объединении с предыдущей цепочкой равенств это означает, что компактный оператор $\mathscr{T}_{w}\left(a x, v_{K}\right)$ совпадает с $a \diamond \sigma_{K}(x)$ или, что то же самое, с $\varsigma\left(\left(\sigma_{K}\right)_{\infty}(a x)\right)$, где, как мы помним, $\left(\sigma_{K}\right)_{\infty}(a x) \in \mathscr{K}[\mathscr{K}]$. Следовательно, с учетом линейности соответствующих отображений для каждого $u \in \mathscr{K}[\mathscr{K}(H)]$ выполнено $\mathscr{T}_{w}\left(u, v_{K}\right)=\varsigma\left(\left(\sigma_{K}\right)_{\infty}(u)\right)$. Но $\varsigma: \mathscr{B}(L \dot{\otimes} L) \rightarrow \mathscr{B}-$ изометрический изоморфизм. Поэтому

$$
\left\|\mathscr{T}_{w}\left(u, v_{K}\right)\right\|=\left\|\left(\sigma_{K}\right)_{\infty}(u)\right\| .
$$

А теперь напомним, что $\mathscr{K}(H)$ и $\mathscr{K}$ взяты с конкретными квантовыми нормами, и рассмотрим диаграмму

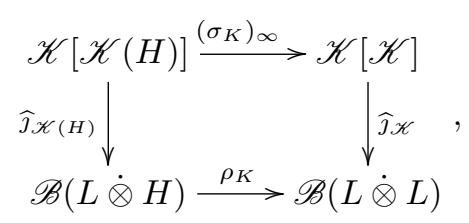

в которой $\widehat{\jmath}_{(\cdot)}$ - естественные вложения из $\S 1$, а $\rho_{K}$ переводит оператор $u$ в композицию $\left(\mathbf{1} \dot{\otimes} S_{K}\right) u\left(\mathbf{1} \dot{\otimes} S_{K}^{*}\right)$. Эта диаграмма, как легко проверить на элементарных тензорах, коммутативна. Поэтому для каждого $u \in \mathscr{K}[\mathscr{K}(H)]$ при его отождествлении с оператором $j_{\mathscr{K}(H)}(u)$ выполнено

$$
\left\|\left(\sigma_{K}\right)_{\infty}(u)\right\|=\left\|\left(\mathbf{1} \dot{\otimes} S_{K}\right) u\left(\mathbf{1} \dot{\otimes} S_{K}^{*}\right)\right\| .
$$


Следовательно, с учетом равенства (13)

$$
\left\|\mathscr{T}_{w}\left(u, v_{K}\right)\right\|=\left\|\left(\mathbf{1} \dot{\otimes} S_{K}\right) u\left(\mathbf{1} \dot{\otimes} S_{K}^{*}\right)\right\| .
$$

Это равенство вместе с (11) из теоремы $1^{\prime \prime}$ и тем, что $\left\|\mathbf{1} \dot{\otimes} S_{K}\right\|=\left\|\mathbf{1} \dot{\otimes} S_{K}^{*}\right\|=1$, влечет $\left\|v_{K}\right\| \leqslant 1$ для всех $K$.

Далее, $\mathbf{1} \dot{\otimes} S_{K}$ - частично изометрический оператор с начальным проектором $1 \dot{\otimes} P_{K}$. Отсюда ясно, что

$$
\left\|\left(\mathbf{1} \dot{\otimes} S_{K}\right) u\left(\mathbf{1} \dot{\otimes} S_{K}^{*}\right)\right\|=\left\|\left(\mathbf{1} \dot{\otimes} P_{K}\right) u\left(\mathbf{1} \dot{\otimes} P_{K}\right)\right\| .
$$

Но, как хорошо известно, $P_{K}, K \in\{K\},-$ ограниченная аппроксимативная единица в $\mathscr{K}(H)$. Отсюда очевидным образом вытекает, что

$$
\lim _{K}\left\|\left(\mathbf{1} \dot{\otimes} P_{K}\right) u\left(\mathbf{1} \dot{\otimes} P_{K}\right)\right\|=\|u\|
$$

для всех $u \in \mathscr{K}[\mathscr{K}(H)]$. Вместе с равенствами (14) и (15) это дает

$$
\lim _{K}\left\|\mathscr{T}_{w}\left(u, v_{K}\right)\right\|=\|u\| .
$$

Дальше ясно.

Перейдем ко второй классической двойственности с участием пространства $\mathscr{N}(H)$, а именно, $\widetilde{T}: \mathscr{N}(H) \times \mathscr{B}(H):(y, x) \mapsto \operatorname{tr}(y x)$. На основании второй теоремы Шэттена-фон Нейманна она порождает изометрический изоморфизм $\widetilde{\mathscr{T}^{\mathscr{B}}(H)}: \mathscr{B}(H) \rightarrow \mathscr{N}(H)^{*}$, позволяющий отождествлять $\mathscr{B}(H)$ с сопряженным нормированным пространством к $\mathscr{N}(H)$. Покажем, что и в этом случае происходит отождествление соответствующих квантовых пространств.

ТЕОРема 5. Пусть $\mathscr{B}(H)$ рассмотрено как конкретное квантовое пространство, а $\mathscr{N}(H)^{*}$ - как квантовое сопряженное $\kappa$ стандартному квантовому пространству $\mathscr{N}(H)$. Тогда $\widetilde{\mathscr{T}}^{\mathscr{B}}(H)$ - полностъю изометрический изоморфизм.

ДокАзАтельство. Сперва сделаем несколько предварительных наблюдений. Положим для каждого $K$ (см. выше) $R_{K}^{1}: \mathscr{N}(H) \rightarrow \mathscr{N}(H): y \mapsto y P_{K}$ и $R_{K}^{2}: \mathscr{B}(H) \rightarrow \mathscr{B}(H): x \mapsto P_{K} x$ и рассмотрим для всех $v \in \mathscr{K}[\mathscr{N}(H)]$, $u \in \mathscr{K}[\mathscr{B}(H)]$ элементы $v\left(\mathbf{1} \otimes P_{K}\right):=\left(R_{K}^{1}\right)_{\infty}(v) \in \mathscr{K}[\mathscr{N}(H)]$ и $\left(\mathbf{1} \otimes P_{K}\right) u:=$ $\left(R_{K}^{2}\right)_{\infty}(u) \in \mathscr{K}[\mathscr{B}(H)]$. (Они же, при интерпретации $v, u$ и $\mathbf{1} \otimes P_{K}$ как операторов, действующих в $L \dot{\otimes} H$, суть просто операторные композиции.) Таким образом, для элементарных тензоров введенные элементы имеют вид $(b y)\left(\mathbf{1} \otimes P_{K}\right)=$ $b\left[y P_{K}\right]$ и $\left(\mathbf{1} \otimes P_{K}\right)(a x)=a\left[P_{K} x\right], b, a \in \mathscr{K}, y \in \mathscr{N}(H), x \in \mathscr{B}(H)$. Выделим равенства

$$
\begin{gathered}
\widetilde{\mathscr{T}}_{w}\left(v\left(\mathbf{1} \otimes P_{K}\right), u\right)=\widetilde{\mathscr{T}}_{w}\left(v,\left(\mathbf{1} \otimes P_{K}\right) u\right), \\
\left\|\widetilde{\mathscr{T}}_{w}(u, v)\right\|=\left\|\mathscr{T}_{w}(v, u)\right\|, \quad v \in \mathscr{K}[\mathscr{N}(H)], \quad u \in \mathscr{K}[\mathscr{K}(H)] .
\end{gathered}
$$

Первое равенство легко проверяется на элементарных тензорах, а второе следует из формулы $\widetilde{\mathscr{T}}_{w}(v, u)=\triangle \mathscr{T}_{w}(u, v) \triangle$, которая также, с учетом равенства $(1)$, легко проверяется на элементарных тензорах. 
Теперь покажем, что для тех же $v$ и $u$

$$
\widetilde{\mathscr{T}}_{w}(v, u)=\lim _{K} \widetilde{\mathscr{T}}_{w}\left(v\left(\mathbf{1} \otimes P_{K}\right), u\right) .
$$

Сперва рассмотрим случай $v=b y$ и $u=a x$. Тогда утверждение сводится к доказательству равенства $\operatorname{tr}(y x) b \diamond a=\lim _{K} \operatorname{tr}\left(y P_{K} x\right) b \diamond a$. Но направленность $P_{K}$ является (неограниченной) аппроксимативной единицей в $\mathscr{N}(H)$. (Это хорошо известный факт, легко следующий из приведенного в начале раздела определения ядерной нормы.) Кроме того, следовый функционал на $\mathscr{N}(H)$ ограничен. В объединении оба факта дают то, что $\operatorname{tr}\left(y P_{K} x\right)=\operatorname{tr}\left(x y P_{K}\right)$ стремится $\mathrm{k} \operatorname{tr}(x y)=\operatorname{tr}(y x)$. Тем самым равенства (16) справедливы для элементарных тензоров, а значит, и для их сумм, т.е. в полной общности.

Далее, как мы знаем, $\|\mathscr{T}\|_{w c b}=1$, а $\left\|\left(\mathbf{1} \otimes P_{K}\right) u\right\|, u \in \mathscr{K}[\mathscr{B}(H)]$, - это норма соответствующего оператора в $L \dot{\otimes} H$. Объединяя эти факты с равенством $\left\|\widetilde{\mathscr{T}}_{w}\left(u\left(\mathbf{1} \otimes P_{K}\right), v\right)\right\|=\left\|\mathscr{T}_{w}\left(\left(\mathbf{1} \otimes P_{K}\right) v, u\right)\right\|$ - прямым следствием формул $(16)$, а также с равенством (17), мы получаем, что для всех $v$ и $u$ справедлива оценка

$$
\left\|\widetilde{\mathscr{T}}_{w}(v, u)\right\| \leqslant\|v\|\|u\|
$$

иными словами, $\widetilde{\mathscr{T}}$ (а не только $\mathscr{T})$ - слабо полностью ограниченный биоператор и $\|\widetilde{\mathscr{T}}\|_{w c b} \leqslant 1$.

Перейдем к основной части доказательства. Зафиксируем $u \in \mathscr{K}[\mathscr{B}(H)]$ и положим $\Phi:=\widetilde{\mathscr{T}}_{\infty}^{\mathscr{B}(H)}(u)$. Простая проверка на элементарных тензорах показывает, что

$$
\langle\langle v, \Phi\rangle\rangle=\widetilde{\mathscr{T}}_{w}(v, u)
$$

для всех $v \in \mathscr{K}[\mathscr{N}(H)]$. Отсюда в силу оценки (17) и определения квантового сопряженного пространства мы получаем, что $\|\Phi\| \leqslant\|u\|$. Осталось установить обратное неравенство.

Обратим внимание на очевидное соотношение

$$
\mathscr{T}_{w}\left(u^{\prime}, v\left(\mathbf{1} \otimes P_{K}\right)\right)=\mathscr{T}_{w}\left(\left(\mathbf{1} \otimes P_{K}\right) u^{\prime}, v\right), \quad u^{\prime} \in \mathscr{K}[\mathscr{K}(H)], \quad v \in \mathscr{K}[\mathscr{N}(H)]
$$

(ср. первое из равенств (16)). Из него вытекает, что

$$
\left\|v\left(\mathbf{1} \otimes P_{K}\right)\right\| \leqslant \sup \left\{\left\|\mathscr{T}_{w}\left(\left(\mathbf{1} \otimes P_{K}\right) u^{\prime}, v\right)\right\|: u^{\prime} \in \mathscr{K}[\mathscr{K}(H)],\left\|u^{\prime}\right\| \leqslant 1\right\} .
$$

Поскольку $\mathscr{K}(H)$ - конкретное квантовое пространство, $\left\|\left(\mathbf{1} \otimes P_{K}\right) u^{\prime}\right\| \leqslant\left\|u^{\prime}\right\|$. Отсюда наша верхняя грань не превосходит $\sup \left\{\left\|\mathscr{T}_{w}\left(u^{\prime}, v\right)\right\|: u^{\prime} \in \mathscr{K}[\mathscr{K}(H)]\right.$, $\left.\left\|u^{\prime}\right\| \leqslant 1\right\}$, т.е. $\|v\|$. Это означает, что $\left\|v\left(\mathbf{1} \otimes P_{K}\right)\right\| \leqslant\|v\|$ для всех $v \in \mathscr{K}[\mathscr{N}(H)]$ и $K$. Как следствие этого факта, определения нормы в $\mathscr{K}[\mathscr{B}(H)]^{*}$ и формулы (19) имеем

$$
\|\Phi\| \geqslant \sup \left\{\left\|\widetilde{\mathscr{T}_{w}}\left(v\left(\mathbf{1} \otimes P_{K}\right), u\right)\right\|: v \in \mathscr{K}[\mathscr{N}(H)],\|v\| \leqslant 1\right\} .
$$

Из равенств (16) следует, что последняя верхняя грань равна

$$
\sup \left\{\left\|\mathscr{T}_{w}\left(\left(\mathbf{1} \otimes P_{K}\right) u, v\right)\right\|: v \in \mathscr{K}[\mathscr{N}(H)],\|v\| \leqslant 1\right\} .
$$


Но согласно теореме 4 это число есть норма элемента $\left(\mathbf{1} \otimes P_{K}\right) u \in \mathscr{K}[\mathscr{K}(H)]$, или, что то же самое, норма оператора $\left(\mathbf{1} \dot{\otimes} P_{K}\right) u \in \mathscr{B}(L \dot{\otimes} H)$.

Итак, $\|\Phi\| \geqslant\left\|\left(\mathbf{1} \dot{\otimes} P_{K}\right) u\right\|$ для всех $K$. Но, как известно, норма операторов $\left(\mathbf{1} \dot{\otimes} P_{K}\right) u$ и $u$ есть верхняя грань чисел соответственно $\left|\left\langle\left[\left(\mathbf{1} \otimes P_{K}\right) u\right] \xi, \eta\right\rangle\right|$ и $|\langle u \xi, \eta\rangle|$ по всем $\xi, \eta$ из единичного шара пространства $L \dot{\otimes} H$ и к тому же $\xi=\lim _{K}\left(\mathbf{1} \otimes P_{K}\right) \xi$ для всех $\xi \in L \dot{\otimes} H$. (Последнее легко следует из очевидного равенства $\zeta=\lim _{K} P_{K} \zeta$ для всех $\zeta \in H$.) Это означает, конечно, что

$$
\sup _{K}\left\|\left(\mathbf{1} \dot{\otimes} P_{K}\right) u\right\| \geqslant\|u\|
$$

Дальше ясно.

\section{Список литературы}

[1] E. G. Effros, "Advances in quantized functional analysis", Proceedings of the International Congress of Mathematicians, vol. 1, 2 (Berkeley, CA, 1986), Amer. Math. Soc., Providence, RI, 1987, 906-916.

[2] E. G. Effros, Z.-J. Ruan, Operator spaces, London Math. Soc. Monogr. (N.S.), 23, Clarendon Press, Oxford, 2000.

[3] V. I. Paulsen, Completely bounded maps and operator algebras, Cambridge Univ. Press, Cambridge, 2002.

[4] D. P. Blecher, C. Le Merdy, Operator algebras and their modules - an operator space approach, London Math. Soc. Monogr. (N.S.), 30, Oxford Univ. Press, Oxford, 2004.

[5] G. Pisier, Introduction to operator space theory, Cambridge Univ. Press, Cambridge, 2003.

[6] C. Webster, Matrix compact sets and operator approximation properties, arXiv: math.FA/ 9804093.

[7] C.-K. Ng, From operator spaces to topological bimodules, preprint.

[8] B. Magajna, "The minimal operator module of a Banach module", Proc. Edinb. Math. Soc. (2), 42:1 (1999), 191-208.

[9] C. Pop, Bimodules normés représentables sur les espaces hilbertiens, arXiv: math. OA/ 9807054.

[10] A. Ya. Helemskii, "Tensor products in quantum functional analysis: non-coordinate approach", Topological Algebras and Applications 2005, Amer. Math. Soc., Providence, RI (to appear).

[11] E. G. Effros, Z.-J. Ruan, "A new approach to operator spaces", Canad. Math. Bull., 34 (1991), 329-337.

[12] D. P. Blecher, V. I. Paulsen, "Tensor products of operator spaces", J. Funct. Anal., 99 (1991), 262-292.

[13] А.Я. Хелемский, Лекиии по функциональному анализу, МЦНМО, М., 2004.

[14] C. Le Merdy, "On the duality of operator spaces", Canad. Math. Bull., 38:3 (1995), 334-346.

[15] M. Takesaki, Theory of operator algebras. I, Springer-Verlag, New York, 1979.

\section{А. Я. Хелемский (А. Yа. Helemskiř)}

Московский государственный университет

им. М. В. Ломоносова

E-mail: alexander@helemskii.mccme.ru
Поступила в редакцию 10.11.2005 и 12.07.2006 